Author Copy

\title{
Negative Emotion Enhances Memory for the Sequential Unfolding of a Naturalistic Experience
}

\author{
Deea K Dev*1, Victoria Wardell*1 ${ }^{* 1}$ Katherine J. Checknita ${ }^{1}$, Alessandra A. Te ${ }^{1}$, \\ Aria S. Petrucci ${ }^{1}$, M. Lindy Le ${ }^{1}$, Christopher R. Madan², Daniela J. Palombo ${ }^{1}$ \\ *Authors contributed equally \\ ${ }^{1}$ Department of Psychology, University of British Columbia \\ ${ }^{2}$ School of Psychology, University of Nottingham
}

\section{Author Note}

Corresponding author:

Daniela J. Palombo, Department of Psychology, University of British Columbia, 2136 West Mall, Vancouver, BC, V6T 1Z4, Canada. daniela.palombo@psych.ubc.ca 


\begin{abstract}
The events of our lives unfold across time. When remembering these events, we often reference information about when they occurred and their sequential unfolding. How does negative emotion affect our ability to reconstruct the elements of an event in the correct temporal order? This study explored this question using naturalistic film stimuli. Human participants $(N=276)$ saw video clips varying in emotion (high versus low). Later, participants were asked to reconstruct the events in the encoded order. Participants' temporal-order memory was better in the high- versus low-emotion condition. Free-recall data showed that participants remembered the high-emotion video with greater vividness, though consistency of details did not differ, nor did spontaneous ordering of clips. Our findings shed light on the multifaceted effects of negative emotion on memory, suggesting that highly negative events are reconstructed with greater temporal fidelity when order is a task demand. Theoretical and practical implications are discussed.
\end{abstract}

Keywords. emotion, memory, movie, naturalistic, time 


\section{General Audience Summary}

The act of remembering often involves reconstructing the order in which an event originally occurred. This allows us to form coherent memories that are situated in a context in which we can visualize the unfolding of events across time. We rely upon this aspect of memory not only in day-to-day reminiscing, but also in legal settings, whereby witnesses are asked to recount their memory of how the event in question occurred. Studies, however, have identified that emotion has complex effects on memory, wherein emotion can enhance or impair certain elements of a memory. This effect is less studied for dimensions of memory related to time (i.e., memory for temporal information), particularly that of temporal order. Considering the emotional nature of many of life's important events and those related to court cases and investigations, it is crucial to identify the exact relation between emotion and temporal-order memory. Hence in this study, we sought to elucidate the influence of negative emotion on our ability to reconstruct the temporal order of an event. We examined temporal-order memory for a film excerpt, comparing memory between participants exposed to a high- versus low-emotion cut of the film. We found that higher negative emotionality resulted in enhanced temporal-order memory. In an ancillary free-recall task, we further found that the high-emotion film was remembered with more episodic detail, yet the proportion of accurate details provided and the fidelity of spontaneously provided order information did not differ between emotion conditions. Our results indicate that negative emotion can augment our ability to recall events in an accurate temporal order when order is a task requirement, and in doing so, allows one to recreate a timeline of our salient life events. 


\section{Negative Emotion Enhances Memory for the Sequential Unfolding of a Naturalistic Experience}

Episodic memories allow for the recollection of specific spatiotemporal events and can consist of varying levels of specificity (Tulving, 1972). A robust body of literature indicates that emotion facilitates detailed episodic memory (e.g., Cahill \& McGaugh, 1995; Kensinger \& Corkin, 2003; LaBar \& Cabeza, 2006). This emotional memory enhancement effect has been demonstrated across a range of stimuli and paradigms, for laboratory and real-world memoranda alike (e.g., Ack Baraly, Hot, Davidson, \& Talmi, 2017).

However, the effects of emotion on memory are not uniform. Emotion enhances memory for the "central" emotional content of an event per se, while impairing memory for contextual information (e.g. Kensinger, Garoff-Eaton, \& Schacter, 2007) and associations with centrally presented items, particularly when the emotional content is negative (Madan, Caplan, Lau, \& Fujiwara, 2012; Bisby, Horner, Bush, \& Burgess, 2018; Palombo, Te, Checknita, \& Madan, 2021; Madan, Scott, Kensinger, 2019).

One aspect of context that has received surprisingly little attention in studies of emotional memory is temporal context, or remembering when something occurred. In the present study, we explore how varying emotion impacts the ability to remember temporal aspects of an event. Here, we focus on negative (as opposed to positively valenced) emotional stimuli as a first step. Although both negative and positive emotion have been shown to enhance item memory, the effects of positive emotion on associative and temporal processing are a little less consistent overall, in part due to challenges with manipulating stimulus properties for positive stimuli (for review, see Bowen, Kark, \& Kensinger, 2018; Clewett \& Murty, 2019; Kensinger \& Ford, 2020; Petrucci \& Palombo, 2021). Here, we manipulate negative emotion both in terms of arousal (i.e., how much excitement the stimuli elicit) and valence (i.e., how 
negative—as opposed to neutral—the stimuli are) to classify our stimuli in terms of high- versus low- negative emotionality. ${ }^{1}$

Although studies of emotion and temporal memory are sparse, some evidence suggests that emotion affects temporal memory. For example, studies focusing on temporal duration suggest that negative events in particular are later remembered as being longer in duration than neutral events (a more ambiguous pattern is observed for positive events; reviewed recently in Petrucci \& Palombo, 2021). Less clear are the effects of emotion on memory for the temporal order of occurrences, meaning the chronological sequence of events-the focus of this study. Some evidence suggests that both negative and positive emotion (Huntjens, Wessel, Postma, van Wees-Cieraad, \& de Jong, 2015) or only negative emotion (Maddock \& Frein, 2009) impairs temporal-order memory (also see de Montpellier, Bisby, \& Burgess, 2021 for a similar finding in a study involving only negative stimuli) in tasks involving temporal reconstruction or judgements of order. Free-recall work suggests that emotion disrupts the tendency to recall items in the order they occurred (Talmi, Lohnas, \& Daw, 2019). In contrast, there are studies which show that emotion (in this case, both positive and negative) enhances (Schmidt, Patnaik, \& Kensinger, 2011) or is unrelated to temporal-order memory (Makowski, Sperduti, Nicolas, \& Piolino, 2017). ${ }^{2}$ Taken together, these studies demonstrate ambiguity in the role of emotion in temporal-order memory.

Another paradigm used to study memory for time involves making source memory judgements for sequentially encoded lists, which perhaps represents a coarser appraisal of

\footnotetext{
${ }^{1}$ This approach, namely, to consider both arousal and valence, is common in studies involving emotional pictures (e.g., those that use the International Affective Picture System or Nencki Affective Picture System databases). The terms of high- versus low-emotionality are used in a relative manner in the present study, whereby both measures of emotion were rated below neutral on valence, but 'high' is commensurate with 'low'. In truth, achieving true neutrality of emotion in events is challenging (Izard, 2007).

${ }^{2}$ Palombo et al. (2021) showed no effect of negative emotion on temporal precision, although negative emotion did affect bias (less bias for negative versus neutral stimuli). This study, however, did not probe temporal order.
} 
temporal-order memory (Palombo \& Cocquyt, 2020). Most such studies indicate that emotion improves temporal source memory judgements (e.g., D’Argembeau \& Van der Linden, 2005; Rimmele, Davachi, \& Phelps, 2012; but also see Hennings, Lewis-Peacock, and Dunsmoor, 2021), and this effect is somewhat more consistent for negative stimuli (see Petrucci \& Palombo, 2021). Still, it is unclear whether source memory judgements rely solely upon temporal information, as there may be other modulatory factors influencing task performance. For example, enhanced source memory for emotional items may not be attributed wholly to information drawn from temporal context - a strong binding to the context formed across emotional items within a single block itself could contribute to the enhanced temporal source judgement performance. Nevertheless, given the possibility that such tasks may probe temporal-order memory, these results further contribute to an overall lack of clarity about the influence of negative emotion on temporal-order memory.

To illuminate the role of negative emotion in memory for temporal order, the present study investigates the effects of negative emotion on temporal-order memory using a temporalreconstruction task. We used video clips from a movie as a means to emulate the unfolding of real-life events. The studies reviewed above have largely used static stimuli (images or words) as they afford great experimenter control. Although the latter approach has yielded insights, its ecological validity may be less robust for studying temporal memory. Naturalistic stimuliincluding film, spoken narrative, or virtual reality—can more readily be employed to create sequentially unfolding dynamic events to mimic emotional events experienced in real life, such as witnessing a crime or an accident. By harnessing the utility of naturalistic stimuli, the present study is robustly designed to examine temporal-order memory for an emotional event (with a focus here on negative emotion).

In this study, we edited selected clips from a movie to create two experimental conditions (high- versus low-emotion; confirmed through norming, see below); as noted above 'high' is construed in terms of highly negative and arousing. The movie selected, Pihu 
(Screwvala, Roy Kapur, Jindal, \& Kapri, 2018), is a Hindi film featuring a toddler who is alone in her home with her deceased mother. The clips in the movie depict her exploring her home and range in emotional intensity from mundane to intense (Figure 1). The high-emotion clips were initially selected based on depicted content - specifically, the presence of "shock value", for example a clip showing the deceased mother, and unsettling occurrences, such as the toddler being in potentially dangerous situations (for descriptions of the clips, see Table S1). While the low-emotion clips did feature some degree of negative undertones as the young toddler was pictured alone at home, the clips were absent of strongly arousing and provoking scenes. We examined temporal-order memory by asking participants to arrange the clips into the order they were encoded. Due to the conflicting and sparse literature, we opted not to make specific hypotheses about whether temporal-order memory would be enhanced or impaired in the highversus low-emotion condition.

As a secondary goal, and to align our work with prior literature, we sought to provide a richer understanding of the mnemonic characteristics of emotional events by employing a freerecall narrative approach commonly used in studies of autobiographical memory (e.g., Wardell, Esposito, Madan, \& Palombo, 2021; McKinnon et al., 2015; Diamond \& Levine, 2020). Thus, we also asked whether our conditions elicited differences in other aspects of memory, including the richness and consistency of recall, as well as the extent to which narratives are spontaneously recalled in the correct order.

\section{Methods}

\section{Participants}

Participants in the present study were undergraduate students at the University of British Columbia who were recruited via the UBC Human Subject Pool system and granted course credit in exchange for participation. To be eligible, participants had to be between the ages of 18 and 35, fluent in English, and have normal or corrected-to-normal vision. A norming experiment in an independent group of participants $(N=160)$ was conducted prior to running the main 
experiment (also see Supplemental Materials, including Table S2) to confirm our emotion manipulation. To avoid confusion, we use the nomenclature 'norming' and 'main' experiments when needed. Both experiments in this study took place online due to COVID-19, and were approved by the Behavioural Research Ethics Board at the University of British Columbia.

A total of 373 students registered for the main study. Prior to the study, participants were alerted to the possible triggering content in the video clips related to death, and were given the option to withdraw from the study due to the sensitive material in the videos. Of these participants, 97 were excluded, either because they did not complete the entire study $(N=60)$, opted out of participation due to trigger warnings $(N=19)$, or reported having seen the film previously $(N=18)$. Of the remaining 276 participants included in the study (high-emotion condition: $N=133$; low-emotion condition: $N=143$ ), participants ranged in age from 18 to 35 years old ( $M=20.79$ years, $S D=2.63$ ), though four participants did not provide their age. Gender distributions are included in Supplemental Materials (Table S3).

Given the novelty of our paradigm and online testing within our laboratory, we did not perform an a priori power analysis. Instead, we opted for a convenience sample (i.e., testing as many participants as we could within the academic term, aiming for a minimum of 100 participants in each condition). For comparison, this is notably larger than previous relevant studies that detected significant effects (e.g., Huntjens, et al., 2015, $N=56$; Maddock \& Frein, 2009, $N=38$; Schmidt et al., 2011, $N=24$; all within-subjects designs).

\section{Materials}

Stimulus material for the study was composed of video clips from the Indian film, Pihu (Screwvala et al., 2018). The film is a Hindi drama that depicts a two-year-old toddler, named Pihu, who is alone inside her house with her deceased mother. It can be inferred that her mother died either by suicide or injuries inflicted as a result of domestic violence. The film portrays a sequence of activities in which Pihu engages while unsupervised in the house, ranging from mundane (e.g., brushing her teeth) to alarming (e.g., cooking on a gas stove). 
While all scenes centre on the unsupervised child, the scenes were filmed such that they had no apparent correlation with one another-a scene of Pihu watching television in the den would be followed by a scene of Pihu engaging in an unrelated activity. As such, temporal order can only be generated from watching the film and relying on episodic memory to reconstruct the sequence of clips (particularly after the film was edited, see below). In contrast, a video of a car accident, for example, could be reconstructed temporally based on prior and/or schematic knowledge about how car accidents typically unfold.

As the full film consists of various scenes of differing levels of emotionality, we spliced the film to produce 16 high- and 16 low-emotion clips (see Supplemental Materials for norming). These clips were ordered randomly, deviating from the sequence in which they were shown in the original film. ${ }^{3}$ As shown in Figure 1, the high-emotion clips were compiled into a single, highemotion video stimulus, and the low-emotion clips were compiled into a single, low-emotion video stimulus. Both videos began and ended with the same three neutral clips ("bookend clips") to account for primacy and recency effects that might influence participant performance (see Cahill \& McGaugh, 1995). This resulted in one high-emotion video composed of $22 \mathrm{clips,} \mathrm{and}$ one low-emotion video composed of 22 clips, each of which was $7 \mathrm{~min}$ and $32 \mathrm{sec}$ in duration. Each clip had a $1 \mathrm{sec}$ long fade-out into the subsequent clip.

\footnotetext{
${ }^{3}$ To reduce variability across the conditions apart from the emotional content, the clips included were consistent in that the scenes did not switch within the clip itself, and each centered around a certain action Pihu was doing.
} 
Figure 1. Video Stimuli Paradigm

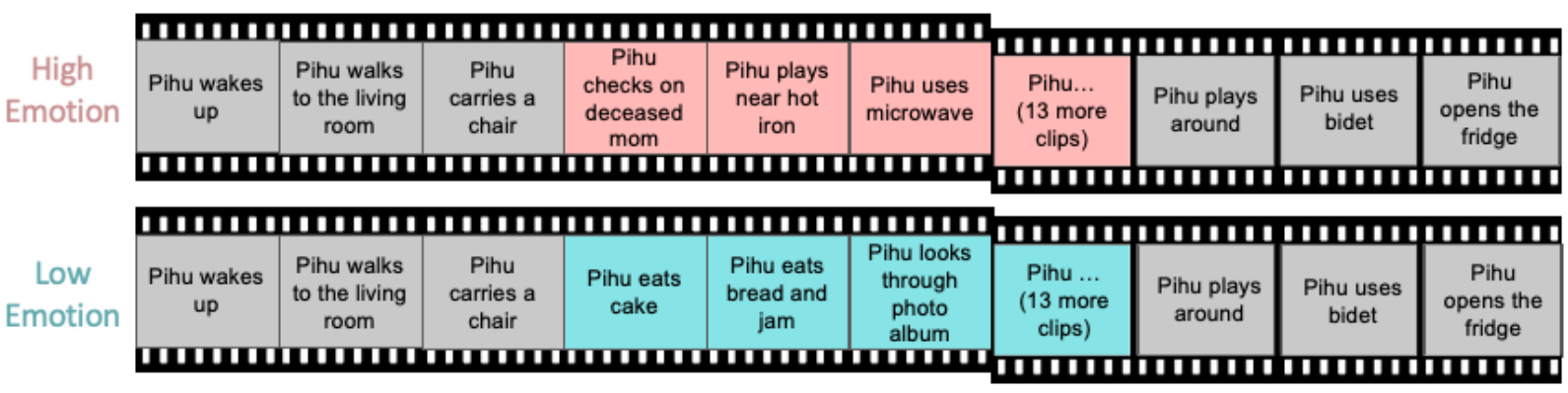

$7 \mathrm{~min}, 32 \mathrm{sec}$

Note. Video stimuli for high-emotion and low-emotion conditions. The start and end "bookend" clips in grey were identical in the two conditions, whereas the middle 16 clips were either high or low in emotion.

\section{Procedure}

First, all participants completed a demographic and health survey in order to characterize the sample, as well as a battery of questionnaires ${ }^{4}$. Participants were then randomly assigned to either the high- or low-emotion condition.

Encoding. Participants watched the video specific to their assigned condition and then rated the overall emotional arousal $(1=$ little to no intensity, $5=$ moderate intensity, $9=$ extreme intensity) and valence ( 1 = very negative, $5=$ neutral, $9=$ very positive) of the video using 9 point Likert scales. Prior to watching the video, participants had been instructed that they would be "watching a video and answering questions about the video." There was no specific detail provided about the nature of the questions. As noted earlier, a separate group of participants in a norming study also provided arousal and valence ratings (see Supplemental Materials; Figure S1), which confirmed that the clips differed in the expected manner with respect to arousal and

\footnotetext{
${ }^{4}$ Measures of clinical symptoms (e.g., anxiety) as well as personality traits (e.g., empathy) were collected, but are beyond the scope of the present paper and not further reported here.
} 
valence (also see Figure 2). Participants were then asked to state how long they thought the duration of the video was, a measure which was included for an exploratory analysis to examine the effects of negative emotion on remembered duration (see Supplementary Materials; for a review, see Petrucci \& Palombo, 2021).

Figure 2. Norming Study - Arousal and Valence Ratings by Condition for Video Clips
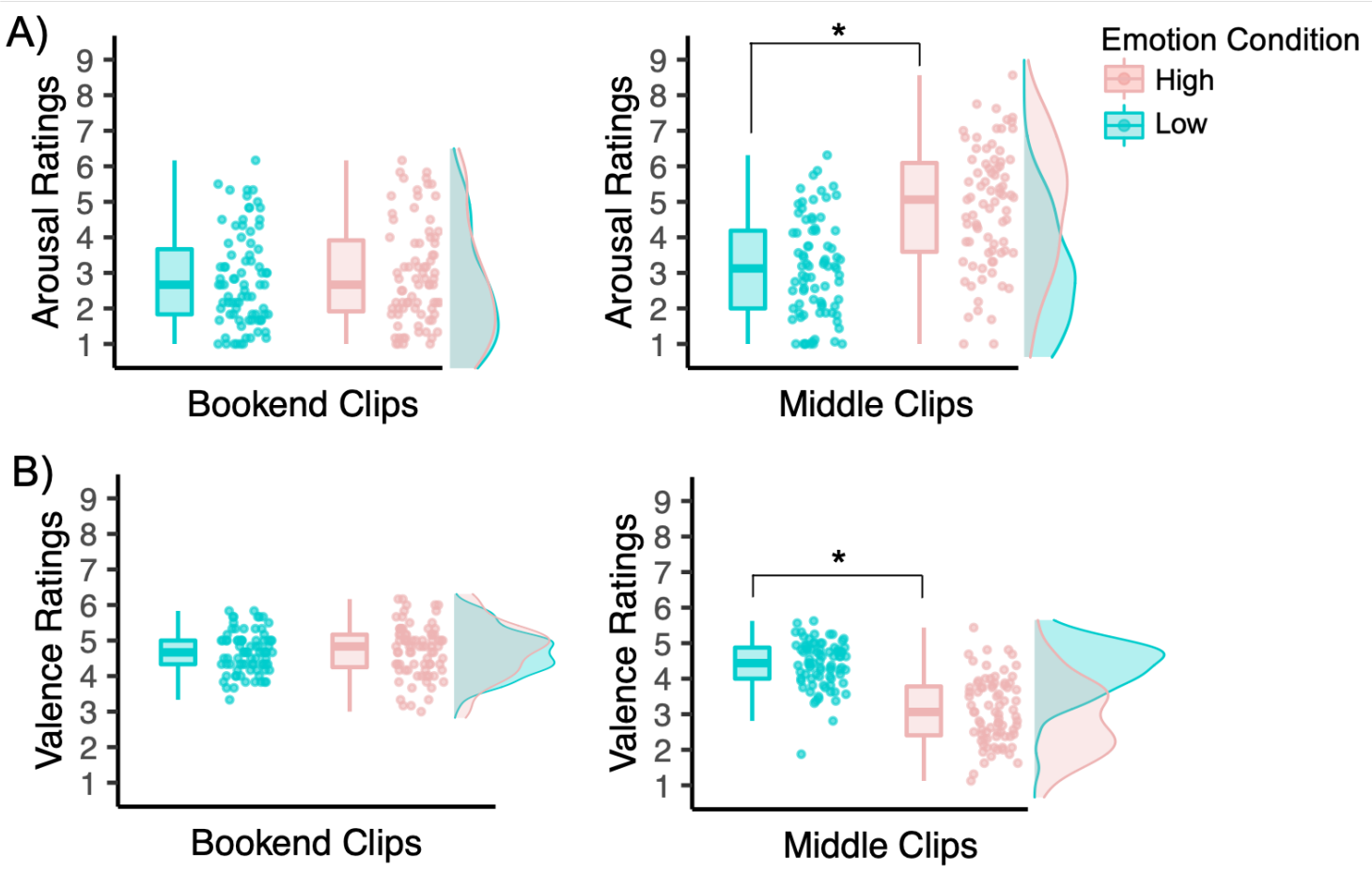

Note. Box plots for A. arousal (upper) and B. valence (lower) ratings for bookend (clips 1-3 and 20-22) and middle clips (clips 4-19) and across high- and low-emotion video conditions in the norming study. For all plots, the centre line on the boxplot = median; the points represent individual participant data; the density plots show the smoothed distribution of the data (also see Supplemental Materials).

Free Recall. Next, participants were asked to type out everything they remembered from the video in as much detail as possible. There were no time restrictions for completing this task. An example description of the Disney Pixar film Up (Rivera \& Docter, 2009) was provided to 
ensure participants understood the types of details we were requesting of them to provide (see Supplemental Materials).

Temporal-Order Reconstruction. Finally, participants were presented with an orderreconstruction task, for which they were asked to arrange still images of the clips (screenshots) into the order they recalled the clip appearing in the video. These stills were representative of the content of the clip such that the participant could identify what the clip was about just by looking at the still image (e.g., the high-emotion clip where Pihu is trapped in the refrigerator is represented by a front angle shot of her sitting inside the refrigerator; the low-emotion clip where Pihu uses the toilet is represented by a still of an overhead shot of Pihu getting on the toilet). The three neutral clips at the start and end of each video were excluded from this task. To decrease task difficulty, the middle clips were presented in two separate blocks of eight clips each. The clips were first sequenced in a random order in these two blocks, then manipulated to ensure that the scenes across each block were well matched in individual distance, mean distance, and mean recency.

Distance was computed as how far apart each scene (corresponding to the respective still shown in the task) was from the scene which chronologically preceded it in the video. To provide a specific example, if a block presents still shots of clips ordered $8,5,10,4$, we compute the distance between clips 4 and 5 , clips 5 and 8 , and clips 8 and 10 . This yields distance scores of 1,3 , and 2 respectively. Recency was computed as how far apart each scene (corresponding to the respective still shown in the task) was from the very first clip shown in the video. Going back to the example (clips 8, 5, 10,4), the recency scores for these clips would be 7, 4, 9 and 3 respectively. To attenuate order effects, the two blocks were counterbalanced across participants.

Debrief. At the end of the study, participants were asked if they were familiar with the film prior to the study and if they understood Hindi, as there was some, albeit minimal, Hindi dialogue in both the high- and low-emotion videos. Participants were then debriefed. 


\section{Data Analysis}

For all relevant variables, we assessed normality. Violations were present for all variables (e.g., the Shapiro-Wilk test indicated a deviation from normality; there were also varying degrees of skewing apparent in histograms). Although ANOVA is often robust to violations of normality in large sample sizes with approximately equal samples, we nonetheless opt to report only non-parametric tests throughout the paper (n.b., the pattern of results did not change when comparing a parametric versus non-parametric approach for all variables).

Temporal-Order Reconstruction. Temporal-order memory scores were computed for each participant by calculating the Spearman rank-order correlation $(\rho)$ between the correct order and the recalled order for each block of the temporal memory task and averaging them. The scores ranged between -1 and 1 , with 1 indicating the recalled order was entirely correct in both blocks. Relative and absolute order scores were also computed, wherein all constituent pairwise comparisons that underlie the order reconstructions were evaluated (see Supplementary Materials). For these latter scoring schemes, computations were likewise made separately for each block and the final score for each participant was calculated as an average of the values from the two blocks. Because temporal-order memory scores calculated from these three methods were highly correlated within each condition, we only report the Spearman correlation in our analysis of memory performance. The mean Spearman correlation coefficients were Fisher Z-transformed for each participant prior to comparing the groups statistically.

Free Recall. Data from the free-recall task was scored to assess the accuracy and temporal order of the recalled memory. We used a procedure modified from the Autobiographical Interview (Al; Levine, Svoboda, Hay, Winocur, \& Moscovitch, 2002), to parse narrative recalls into details based on the information provided.

Episodic Vividness. Recalls were assigned an episodic richness rating by the experimenter. Episodic richness was assessed on a scale of 0 to 6 , based on the richness and specificity with which the recall was described (see Levine et al., 2002). Per the Al protocol, the 
episodic richness rating measures the degree to which the participants evoked an impression of re-experiencing and recreated the contextual perceptual, emotional, and cognitive context.

Episodic Details. To assess the number of episodic details recalled, we segmented recalls into informational clauses, in accordance with the Al procedure. Details directly referencing an occurrence in the film were considered episodic as they were specific to that singular event. Details not related to the content of the film per se were considered 'other', such as metacognitions or editorializations (e.g., I don't really remember what happened), in line with the Al protocol (Levine et al., 2002).

Accuracy. Episodic details were tagged based on their accuracy (see Figure 3). Tags identified each detail as belonging to one of three categories: (1) consistent with an occurrence in the film, (2) reminiscent of an occurrence in the film, or (3) errors of commissions (for similar approaches, see Cohn-Sheehy et al., 2020; Orbach, Lamb, La Rooy, \& Pipe, 2012). Accuracy was assessed by comparing the proportion of consistent details out of the total number of episodic details recalled (summation of consistent, committed, and reminiscent details). For the accuracy analysis, we included only participants who had a minimum of five episodic details in their free-recall narratives, yielding the exclusion of four participants and a remaining sample of 272 (low-emotion: $N=140$; high-emotion: $N=132$ ). Commissions were included in the calculation of the total number of details recalled but were not analyzed separately due to a floor effect, with $88.60 \%$ of recalls containing no more than one committed detail in this sample.

Figure 3. Example of Scored Free Recall 
$/ 5 /$

...The girl began playing with a balloon /CONS/ while dangerously close to an iron /CONS/ which appeared

to be on /CONS/! Then the scene changes to her on a balcony /CONS/, standing really close to the railing /8/

/CONS/ trying to grab her doll /CONS/ with her foot/CONS/. The doll fell down /CONS/ and you could see $/ 6 /$

how high the drop was /CONS/. The scene changes and the little girl is microwaving pita bread /CONS/.

Then she's getting into the fridge /CONS/ and try to shut it a few times unsuccessfully /CONS/ before it $/ 12 /$

shuts /CONS/, with her inside /CONS/. Then something explodes /CONS/ near a window /CONS/ and bits

of glass fall onto the girl /COM/ while she cries /CONS/ at the bottom of the stairs /CONS/...

Note. Numbers highlighted in yellow denote the temporal order of corresponding clips. Green and blue highlights denote accuracy of details recalled, with '/CONS/' marking details consistent with the film, and '/COM/' marking errors of commissions. See Supplementary Materials for examples of free recall narratives with high versus low detail and episodic richness ratings (Figure S2).

Temporal Clustering. To compute a temporal clustering score (which refers to the tendency to recall nearby items successively; Polyn, Norman, \& Kahana, 2009) from the freerecall data, we further identified which of the 22 clips the recalled details corresponded to. Each clip was assigned a number that corresponded to the order the clip was shown in the video. Recalled details were tagged with the clip number to denote how temporal order manifested during the free recall (for a similar approach, see Diamond \& Levine, 2020; also see Polyn et al. 2009). See Figure 3 for a scored recall example (also see Supplementary Materials). Using the outputted temporal tags, we were able to quantify each transition by computing the proportion of possible transitions that are greater than the transition in question (with no consideration of transition direction). Note that an average score is taken for tied transitions, as per Polyn et al. 
(2009). The score for each transition is averaged to yield a total score. A score of 1 indicates that the participant always made the shortest possible transition, whereas a score of .5 suggests chance-level performance (i.e., no temporal clustering). To provide a specific example, imagine a participant recalled four items from a study list $(1,2,3,4)$ in the order: $3,1,2,4$. The first transition is from the 3rd to the 1st item, yielding a 'jump' of 2, against possible transitions to the 2nd and 4th items, which would involve jumps of 1 . Accordingly, the observed transition would be a score of 0 since it was the furthest possible jump. The same logic then applies to the next transition from the first to the 2nd item and so on (See Figure S3 for an example transcript and temporal order calculation). This analysis focused only on the 16 middle clips in accordance with the temporal-order reconstruction task. For this analysis we included only participants who had a minimum of five temporal tags (as in Diamond \& Levine, 2020), yielding exclusions of 62 participants and a remaining sample of 214 (low-emotion: $N=108$; high-emotion: $N=105$ ).

Interrater Reliability. All scoring of free-recall data was completed by a primary scorer (V.W.). To confirm reliability, a random subset of $10 \%$ of the recalls for both emotion conditions were scored by a secondary scorer (L.L.) A two-way intraclass correlation (ICC) analysis confirmed excellent agreement between the two scorers, with Cronbach's alpha scores ranging from .82 to .99 (see Table S4). All detail tagging for scoring was done electronically to allow for the automatic summation of scored tags for analysis (see Wardell et al., 2021). L.L. was not aware of the hypotheses of the study (i.e., masked scoring). Notably, as recall depicted condition specific scenes, it was not possible for either scorer to be masked to experimental condition. However, given the very high reliability between scorers, even when stratifying by emotion condition, it is unlikely that problematic biases influenced results (see Table S4). ${ }^{5}$

\section{Results}

\footnotetext{
${ }^{5}$ We further compared the word count of recalls between emotion conditions for a more objective, albeit less nuanced measure of detail production. Recalls from the high-emotion condition contained more words $(M d n=186)$ than the low-emotion condition $(M d n=154 ; U=11352.00, Z=-2.78, p=.005, r=$ -.17; see Supplementary Materials).
} 


\section{Manipulation Checks.}

Arousal Ratings. A Mann-Whitney $U$ test was also conducted to determine if there was a difference in arousal ratings between the high- and low-emotion conditions. Results showed that, as in the norming sample (see Supplementary Materials), participants rated the video as more arousing in the high-emotion $(M d n=7)$ versus low-emotion condition $(M d n=5 ; U=$ 12498.00, $Z=-4.56, p<.001, r=-.27$; Figure 4A). Ancillary analyses confirmed that there were no differences in arousal ratings between non-Hindi and Hindi speakers (see Table S5).

Valence Ratings. A Mann-Whitney $\mathrm{U}$ test was conducted to determine if there was a difference in valence ratings between the high- and low-emotion conditions. Results showed that, as in the norming sample (see Supplementary Materials), participants rated the video as more negative in the high-emotion $(M d n=2)$ versus low-emotion condition $(M d n=3 ; U=$ 4825.00, $Z=-7.23, p<.001, r=-.44 ;$ Figure $4 \mathrm{~A})$. Ancillary analyses confirmed that there were no differences in valence ratings between non-Hindi and Hindi speakers (see Table S5).

Figure 4. Arousal and Valence Ratings, and Fisher Z Correlation Scores by Condition

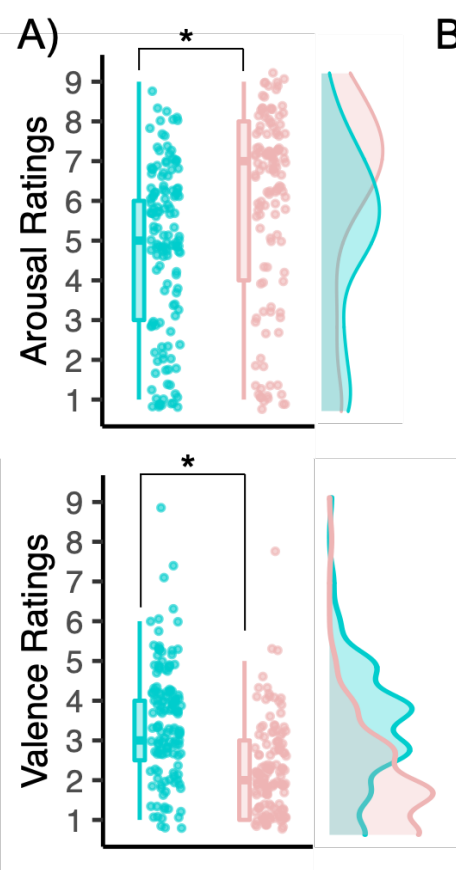

B)

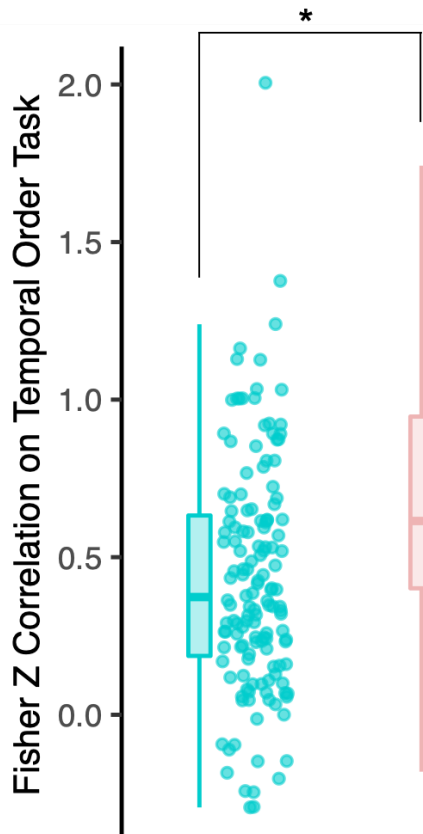

Emotion Condition

High

Low 
Note. Boxplots for the high- and low-emotion video conditions depicting A. arousal (upper left) and valence (lower left) ratings for the main study (see Figure 1 and Supplementary Materials for norming data) and B. Fisher Z transformed rank correlation scores for the temporal-order reconstruction task. For all plots, the centre line on the boxplot $=$ median; the points represent individual participant data; the density plots show the smoothed distribution of the data.

\section{Memory Tasks.}

Given our primary interest was in temporal-order memory, we present the results from that task first.

Temporal-Order Reconstruction. A Mann-Whitney U revealed that the Fisher Z transformed rank correlation scores derived from the temporal-order reconstruction task were greater in the high-emotion condition $(M d n=0.62)$ than the low-emotion condition $(M d n=0.37$; $U=12554.00, Z=-4.59, p<.001, r=-.28$; Figure 4B). This indicates that the participants in the high-emotion condition were more accurate at ranking the order of the film scenes in this task.

Free Recall. Next, we turn to our freerecall task. We analyzed differences between the high- and low-emotion conditions again using Mann-Whitney $U$ tests.

Episodic Vividness. Episodic richness ratings (assigned by the experimenter) of participants' recalls were higher in the high-emotion condition $(M d n=4.00)$ than the lowemotion condition $(M d n=4.00 ; U=11686.00, Z=-3.36, p<.001, r=-.20$; Figure 5A)

Details. The total number of details provided by participants during free recall was greater in the high-emotion condition $(M d n=33.00)$ than the low-emotion condition, $(M d n=29.00 ; U=11520.00, Z=-3.03, p=.002, r=-.18 ;$ not shown $)$. Production of episodic details per se was greater for the high-emotion condition $(M d n=33.00)$ than the low-emotion 
condition $(M d n=29.00 ; U=11539.00, Z=-3.06, p=.002, r=-.18$; see Figure 5B). Indeed, participants mostly provided episodic content overall, so these variables are largely redundant.

Figure 5. Free-Recall Analyses
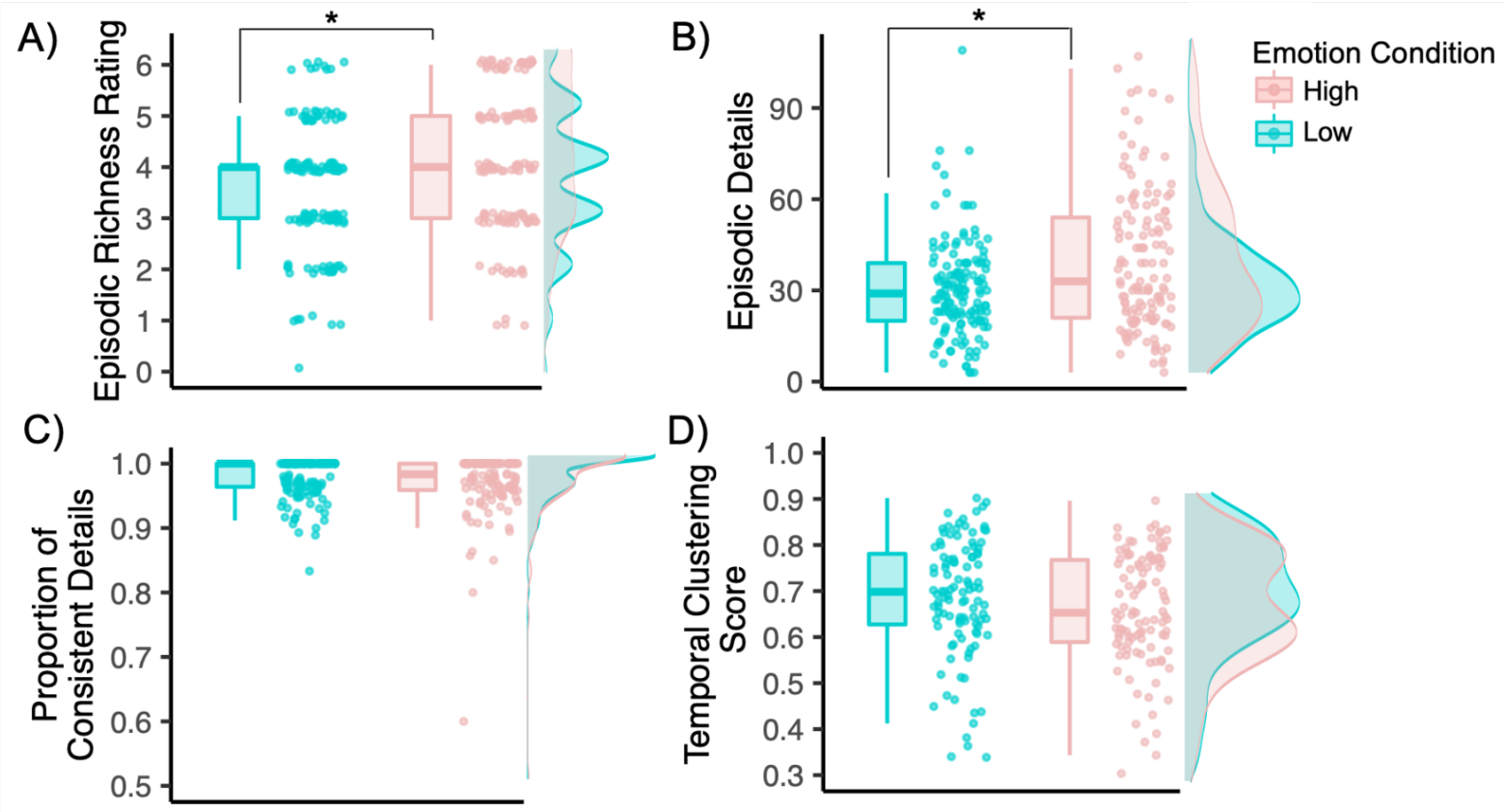

Note. A. Episodic richness ratings; B. total episodic details; C. proportion of consistent details; D. temporal clustering scores. For all plots, the centre line on the boxplot = median; the points represent individual participant data; the density plots show the smoothed distribution of the data.

Accuracy. The accuracy of the details (i.e., the number of consistent versus total episodic details) provided did not differ between high-emotion $(M d n=0.98)$ and low-emotion $(M d n=1.00 ; U=8539.00, Z=-1.16, p=.245, r=-.07)$ conditions. Thus, although participants remembered more details from the high-emotion condition, when they did recall a detail, it tended to be accurate in both conditions (see Figure 5C). 
Temporal Clustering. No difference in temporal clustering was observed within the free-recall narratives between high-emotion $(M d n=0.65)$ and low-emotion conditions $(M d n=0.70 ; U=4883.50, Z=-1.75, p=.080, r=-.12$; Figure 5D). Figure 6 depicts the proportion of recall by clip, for illustrative purposes (also). Thus, emotion did not affect participants' ability to successively recall temporally proximal items in free recall.

Figure 6. Proportion of participants who recalled each clip.
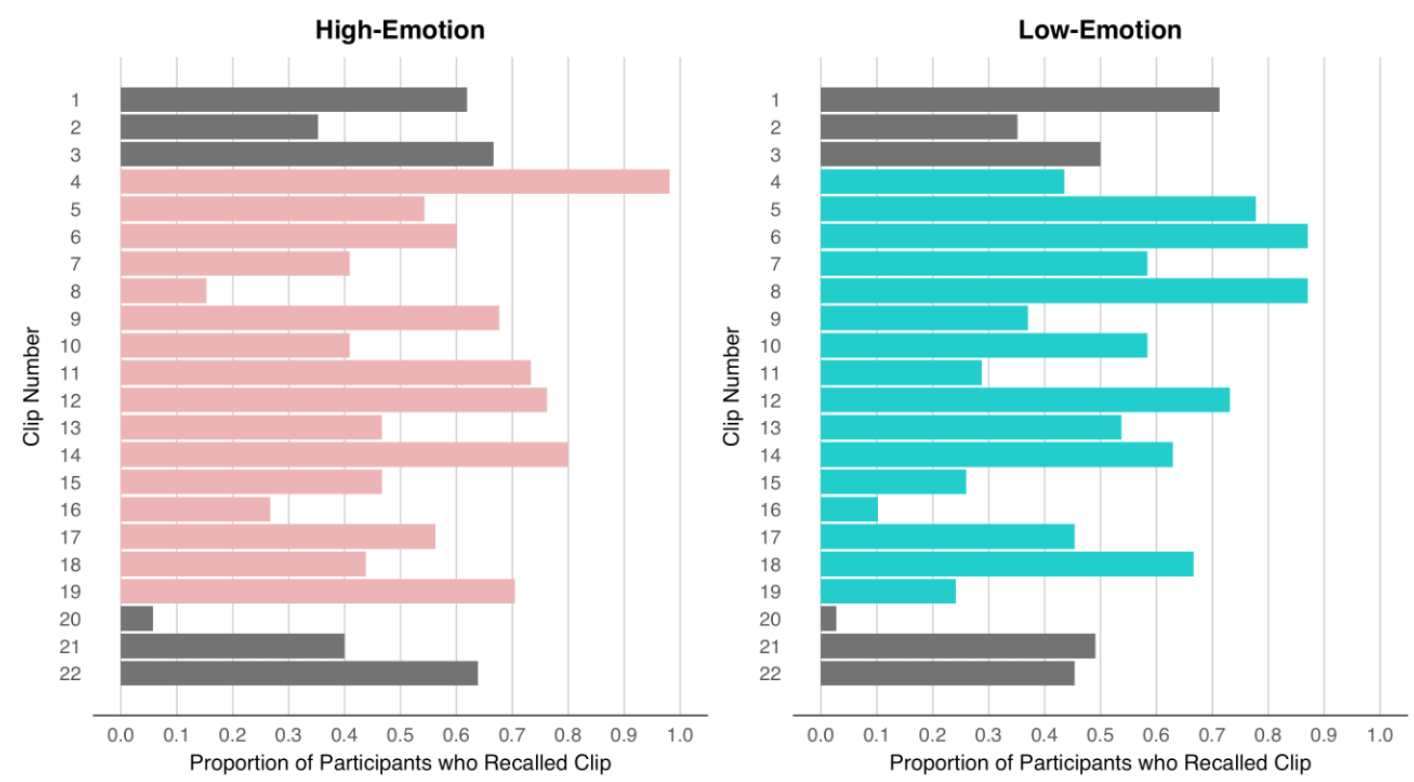

Note. Grey bars mark the six identical bookend neutral clips that started and ended the two videos. See Supplementary Materials for a brief description of each clip (Table S1).

\section{Discussion}

We investigated the influence of negative emotion on temporal-order memory using a naturalistic memory paradigm involving film content. Our study produced four main findings. First, temporal-order memory was better for highly emotional content than that of low emotion. This suggests that highly negative events can be reconstructed with greater temporal fidelity than less emotional events when order is a task demand. Second, greater emotion enhanced the quality of free recall, including the number of details and level of episodic vividness. Third, 
the level of emotion did not affect the proportion of accurate recalled details. Fourth, greater emotion did not affect temporal clustering in free recall. These results are discussed below.

\section{Temporal-Order Reconstruction}

Our finding that negative emotion augments temporal-order memory (in the temporal reconstruction task) adds to a small literature examining the intersection of emotion, time, and memory. Whereas studies using a temporal source-memory paradigm (which may measure temporal-order memory on a coarse timescale), suggest that emotion enhances order memory, studies implementing temporal order judgements are equivocal, leaning more towards an impairment (e.g., Huntjens et al., 2015; Maddock \& Frein, 2009, also see de Montpellier et al., 2021; but see Schmidt et al., 2011). In considering our findings, we speculate that the nature of the stimuli may play an important role (Petrucci \& Palombo, 2021). In general, studies of temporal-order memory have employed static and arbitrary laboratory items presented sequentially. In contrast, our video stimuli more closely mimics an unfolding event (also see Makowski et al., 2017). Temporal-binding mechanisms may be more pertinent when watching a film, due to associations that can be made between composite clips tied by a common context (in this study, the unsupervised toddler), as compared to unrelated pictures or words. Suggestive of this notion, in a prior study, Schmidt et al. (2011) asked participants to chronologically order images that were encoded sequentially on a neutral background; temporal-order memory was better for high versus low arousal images. Critically, participants were asked to form a story about the images, which may have promoted imagery of an unfolding sequence, making it more akin to our paradigm. Unfolding experiences with emotional content—particularly those devoid of a singular attentional magnet—may promote stronger temporal binding to build a causal or predictive model as the details are perceived as goal relevant (Palombo \& Cocquyt, 2020; Levine \& Edelstein, 2010; also see Hoerl et al., 2020). Put another way, when there is more 'at stake', as in emotional situations, it may be prudent to determine the relation among events. 
Mechanistically, augmented temporal binding may be due to greater attention at encoding, as attention is known to be an important explanatory factor behind emotional effects

on memory (Mackay et al., 2004). Indeed, arousal is known to usurp perceptual binding through increasing the selectivity of attention (Mather, 2007; Talmi, Anderson, Riggs, Caplan, \& Moscovitch, 2008; Todd, Miskovic, Chikazoe, \& Anderson, 2020). An alternative, albeit not mutually exclusive, mechanism is that the strong source context imbued by emotional experiences facilitate the reinstatement of encoded temporal context (Talmi et al., 2019; Palombo \& Cocquyt, 2020). Relevant to this idea, recently Clewett and colleagues showed that reduced trial-by-trial variability in pupil response (diameter) across a sequence of items - a proxy for changes in arousal - was associated with better temporal-order memory for those items (Clewett, Gasser \& Davachi, 2020). This finding supports the idea that arousal, as a stable source context, facilitates integration of sequential content. It will be interesting to examine temporal-order memory for experiences where emotional arousal ebbs and flows (i.e., where there is instability in the emotional context).

\section{Free Recall}

Consistent with a host of other studies (reviewed in Ack Baraly et al., 2017), the highemotion video was remembered with more detail than the low-emotion video in free recall. This accords with other work demonstrating that memory is enhanced for emotional content (Hamann, 2001). For example, Hulse, Allan, Memon, and Read (2007) asked participants to watch either a negative or a neutral video; participants in the negative condition had better memory both for the 'central' story as well as 'peripheral' details (also see Laney, Campbell, Heuer, \& Reisberg, 2004). Still, other studies demonstrate trade-off effects, wherein emotion boosts recollection for details central or goal-relevant to the event but reduces memory for peripheral content (Kensinger et al., 2007; Rimmele, Davachi, Petrov, Dougal, \& Phelps 2011; Mather \& Sutherland, 2011). Yet, trade-off effects are less often observed in studies which lack a salient attentional magnet (Levine \& Edelstein, 2010). 
While we found that free recall of the high-emotion film was more detailed, emotion did not affect the accuracy of details that were recalled. Differences in conditions can best be characterized as errors in omission in the low-emotion condition. Prior work demonstrates that, both immediately (Evans \& Fisher, 2011) and after a delay (Diamond, Armson, \& Levine, 2020), individuals tend not to prioritize accuracy over detail in recall. Notably, such studies have not directly explored emotion. Though here we find no emotion effect with a short retention interval, differences in consistency may emerge over longer delays (see Kensinger \& Schacter, 2006). On the other hand, other work suggests that accuracy differences might be more prominent with more pronounced manipulations of valence (e.g., comparing negative to positive stimuli). According to the 'affect-as-information' theory proposed by Clore et al. (2001), the valence of an event plays a key role in providing evaluative information that can directly influence cognitive processes such as memory. More specifically, positive valence tends to promote relational processing between items which results in a higher chance of errors in memory, whereas negative valence leads to detailed and item-specific processing that promotes more accurate memory encoding processes (Clore \& Storebeck, 2006). Several autobiographical memory studies have supported this framework, wherein these studies show that accuracy for an identical event was greater for individuals who perceived it negatively compared to those who did positively (e.g., Bohn \& Bernsten, 2007; Kensinger \& Schacter, 2006b; Levine \& Bluck, 2004). ${ }^{6}$ Considering that our study utilized only stimuli of (high versus low) negative valence, it is possible that our manipulation may not have been potent enough to quell the influence of negative valence in promoting accurate memory for details, even if it did produce other mnemonic effects in our study.

We did not observe condition differences in temporal clustering in free recall. This null finding is somewhat surprising, given the observed emotional enhancement in the temporal-

\footnotetext{
${ }^{6}$ We note that the extent to which such a pattern emerges likely depends on the goal state elicited by the positive or negative situation at hand (also see Murty \& Adcock, 2017; Levine \& Edelstein, 2010).
} 
order reconstruction task. However, order was not a task demand in the free-recall task, and this may account for the different pattern of results. That is, it is possible that the 'boost' observed for temporal memory in the temporal-order reconstruction task is due to the emphasis placed on order but when recalling events naturally, individuals do not prioritize order information for emotional events to the same extent. Our findings underscore the importance of using different tasks to probe temporal order (also see Petrucci \& Palombo, 2021).

\section{Limitations}

In the present study, we selected evocative elements of the film to include in our highemotion condition; those that possessed "shock value" (e.g., Pihu getting herself into the fridge). It is conceivable that the clips in the high-emotion conditions left the viewer unsettled due to the lack of resolution from one clip to the next (e.g., the viewer does not know if Pihu safely escapes). This could result in greater prediction error in the high-emotion condition, compared to the clips in the low-emotion video that might tie together more smoothly. On the other hand, we note that this was part and parcel to our manipulation of emotion, for if every scene had the same arch, the subsequent clips may be less effective in evoking emotion. Relatedly, our highemotion versus low-emotion clips were likely more novel. This consideration is relevant to emotional memory studies more broadly, as this would also be the case for studies that use image stimuli from, e.g., the Nencki Affective Picture System (Marchewka et al., 2014) database, where the negative images often rely on shock-inducing but less familiar visuals to elicit negative emotions. Moreover, as people are much more likely to encounter low-emotion or neutral events in their daily lives (Holland \& Kensinger, 2010), the role of novelty is also relevant to the sampling of emotional events in autobiographical contexts. ${ }^{7}$ These factors are important to consider in future research.

\footnotetext{
${ }^{7}$ On the flip side, one strength of the Pihu stimuli, is that the clips in each video were thematically related to one another in both conditions, which is not seen in stimuli such as sequential NAPS images. According to Talmi and Moscovitch (2004), high semantic relatedness between items can enhance
} 


\section{Application of Findings}

Our findings not only have relevance in the domain of basic science but they also hold applied significance. In the context of legal proceedings, the accuracy of temporal order information can be crucial in understanding what happened during a crime, the intent of a perpetrator, as well as the veracity of eyewitness testimony. For example, an accurate account of when a perpetrator made a threat relative to committing an assault may speak to premeditation. In terms of eyewitness testimony, an accurate reconstruction of the order in which events occur may be relied upon by the prosecution or defense in determining the outcome of a trial, and may also be considered a measure of witness reliability. Given that crimes (or other events relevant to eyewitness accounts) are often highly emotional in nature, it is of vital importance to first understand how emotion affects temporal-order memory (also see Dahl et al., 2018). Our temporal-order reconstruction findings suggest that emotion experienced during some types of events may lead to more faithful witness reports of the timeline of the event in question, relevant to events involving less emotion. Nonetheless, it is important to keep in mind that temporal estimates, even in our high-emotion condition, were far from perfect

In addition, our temporal-clustering analysis suggests that, irrespective of how emotional an event is, free recall of events is unlikely to be a highly faithful reconstruction of the timeline, even if well above chance. This is potentially relevant to the legal system in that freely recalled eyewitness accounts of crimes may not provide an accurate representation of the order in which the crime actually occurred (and could even contaminate subsequent recall; although not assessed here). Still, since order was not a task demand, it will be important to follow up on this work using a free-recall task emphasizing sequential recall, such as those used during witness interviewing.

\section{Future Directions and Conclusion}

memory for emotional material and may account for some of the emotional memory (item) effects observed in the literature. 
Our findings make a novel contribution to the literature by demonstrating the enhancing effects of negative emotion on temporal-order memory, a topic which has scarcely been investigated. The use of more naturalistic stimuli lends credence to our findings, as the stimuli more readily emulate the dynamic nature of behaviours and stimuli in the real world relative to more common laboratory tasks. In doing so, we provide a bridge between two siloed literatures, one investigating the basic science of emotional memory, and another considering memory in the context of eyewitness testimony. Indeed, although the present study did not aim to assess temporal-order memory of eyewitness testimony specifically, our findings nevertheless provide important and relevant insight into how emotional events are later reconstructed from memory. Future research is needed to determine whether these effects generalize to eyewitness testimony per se. Such research will be an important first step for ultimately understanding how to maximize order accuracy of eyewitness testimony (see Gasper, Roy, \& Flowe, 2019). Finally, future research should expand on this work by determining whether these findings generalize to other types of negative emotional experiences (e.g., fear, disgust, anger) or even positive events (e.g., happiness, surprise) (Tsikandilakis, Bali, Derrfuss, \& Chapman, 2020). 


\section{Data Availability Statement}

De-identified data is available through OSF (https://osf.io/jw7e6/). Study materials and analytic code will be made available upon reasonable request. 


\section{References}

Ack Baraly, K., Hot, P., Davidson, P., \& Talmi, D. (2017). How emotional arousal enhances episodic memory. In J. H. Byrne (Ed.), Learning and Memory: A Comprehensive Reference (2nd ed.). Elsevier BV.

Bisby, J. A., Horner, A. J., Bush, D., \& Burgess, N. (2018). Negative emotional content disrupts the coherence of episodic memories. Journal of Experimental Psychology: General, 147(2), 243. https://doi.org/10.1037/xge0000356

Bohn, A., \& Berntsen, D. (2007). Pleasantness bias in Flashbulb Memories: Positive and negative flashbulb memories of the fall of the Berlin Wall among East and West Germans. Memory \& Cognition, 35(3), 565-577. https://doi.org/10.3758/bf03193295

Bowen, H. J., Kark, S. M., \& Kensinger, E. A. (2018). NEVER forget: Negative emotional valence enhances recapitulation. Psychonomic Bulletin \& Review, 25(3), 870-891. https://doi.org/10.3758/s13423-017-1313-9

Cahill, L., \& McGaugh, J. L. (1995). A novel demonstration of enhanced memory associated with emotional arousal. Consciousness and Cognition, 4(4), 410-421. https://doi.org/10.1006/ccog.1995.1048

Clark, I. A., Holmes, E. A., Woolrich, M. W., \& Mackay, C. E. (2016). Intrusive memories to traumatic footage: The neural basis of their encoding and involuntary recall. Psychological Medicine, 46(3), 505-518. https://doi.org/10.1017/S0033291715002007

Clewett, D., DuBrow, S., \& Davachi, L. (2019). Transcending time in The brain: How event memories are constructed from experience. Hippocampus, 29(3), 162-183. https://doi.org/10.1002/hipo.23074

Clewett, D., Gasser, C., Davachi, L. (2020). Pupil-linked arousal signals track the temporal organization of events in memory. Nature Communications, 11(1), 4007. https://doi.org/10.1038/s41467-020-17851-9 
Clewett, D., \& Murty, V. P. (2019). Echoes of emotions past: How neuromodulators determine what we recollect. Eneuro, 6(2). ENEURO.0108-18.2019. https://doi.org/10.1523/ENEURO.0108-18.2019

Clore GL, Storbeck J. (2006). Affect as information about liking, efficacy, and importance. In: Forgas J, editor. Hearts and Minds: Affective influences on social cognition and behaviour. (pp. 123-142). New York: Psychology Press.

Clore, G. L., Wyer, R. S., Jr., Dienes, B., Gasper, K., Gohm, C., \& Isbell, L. (2001). Affective feelings as feedback: Some cognitive consequences. In L. L. Martin \& G. L. Clore (Eds.), Theories of mood and cognition: A user's guidebook (pp. 27-62). Lawrence Erlbaum Associates Publishers.

Cohn-Sheehy, B. I., Delarazan, A. I., Reagh, Z. M., Crivelli-Decker, J. E., Zacks, J. M., \& Ranganath, C. (2020). Bridges, not walls: The hippocampus builds narrative memories across distant events. bioRxiv. https://doi.org/10.1101/2020.12.11.422162

Dahl, M., Granér, S., Fransson, P., Bertilsson, J. \& Fredriksson, P. (2018). Analysis of eyewitness testimony in a police shooting with fatal outcome - Manifestations of spatial and temporal distortions, Cogent Psychology, 5, 1487271. https://doi.org/10.1080/23311908.2018.1487271

D'Argembeau, A., \& Van der Linden, M. (2005). Influence of emotion on memory for temporal information. Emotion, 5(4), 503-507. https://doi.org/10.1037/1528-3542.5.4.503

de Montpellier, E., Bisby, J.A.., \& Burgess, N. (2021). Disrupted associative binding and memory coherence for negative events and their relationship with intrusions. Experimental Psychology Society. Poster presentation presented at Experimental Psychology Society, virtual.

Diamond, N. B., Armson, M. J., \& Levine, B. (2020). The truth is out there: Accuracy in recall of verifiable real-world events. Psychological Science, 31(12), 1544-1556. https://doi.org/10.1177/0956797620954812 
Diamond, N. B., \& Levine, B. (2020). Linking detail to temporal structure in naturalistic-event recall. Psychological Science, 31(12), 1557-1572. https://doi.org/10.1177/0956797620958651

Evans, J. R., \& Fisher, R. P. (2011). Eyewitness memory: Balancing the accuracy, precision and quantity of information through metacognitive monitoring and control. Applied Cognitive Psychology, 25(3), 501-508. https://doi.org/10.1002/acp.1722

Gasper, H. L., Roy, M. M., \& Flowe, H. D. (2019). Improving Time Estimation in Witness Memory. Frontiers in Psychology, 10, 1452. https://doi.org/10.3389/fpsyg.2019.01452

Hamann, S. (2001). Cognitive and neural mechanisms of emotional memory. Trends in Cognitive Sciences, 5(9), 394-400. https://doi.org/10.1016/s1364-6613(00)01707-1

Hennings, A. C., Lewis-Peacock, J. A., \& Dunsmoor, J. E. (2021). Emotional learning retroactively enhances item memory but distorts source attribution. Learning \& Memory, 28(6), 178-186. https://doi.org/10.1101//m.053371.120

Hoerl, C., Lorimer, S., McCormack, T., Lagnado, D. A., Blakey, E., Tecwyn, E. C., \& Buehner, M. J. (2020). Temporal binding, causation, and agency: Developing a new theoretical framework. Cognitive Science, 44(5), e12843-n/a. https://doi.org/10.1111/cogs.12843

Holland, A. C., \& Kensinger, E. A. (2010). Emotion and autobiographical memory. Physics of life reviews, 7(1), 88-131. https://doi.org/10.1016/j.plrev.2010.01.006

Hulse, L. M., Allan, K., Memon, A., \& Read, J. D. (2007). Emotional arousal and memory: A test of the poststimulus processing hypothesis. American Journal of Psychology, 120(1), 7390. https://doi.org/10.2307/20445382

Huntjens, R. J. C., Wessel, I., Postma, A., van Wees-Cieraad, R., \& de Jong, P. J. (2015). Binding temporal context in memory: Impact of emotional arousal as a function of state anxiety and state dissociation. The Journal of Nervous and Mental Disease, 203(7), 545-550. https://doi.org/10.1097/NMD.0000000000000325 
Izard, C. E. (2007). Basic emotions, natural kinds, emotion schemas, and a new paradigm. Perspectives on Psychological Science 2(3), 260-280. https://doi.org/10.1111/j.1745$\underline{6916.2007 .00044 . x}$

Kensinger, E. A., \& Corkin, S. (2003). Memory enhancement for emotional words: Are emotional words more vividly remembered than neutral words? Memory \& Cognition, 31(8), 11691180. https://doi.org/10.3758/BF03195800

Kensinger, E. A., Garoff-Eaton, R. J., \& Schacter, D. L. (2007). Effects of emotion on memory specificity: Memory trade-offs elicited by negative visually arousing stimuli. Journal of Memory and Language, 56(4), 575-591. https://doi.org/10.1016/j.jml.2006.05.004

Kensinger, E. A., \& Schacter, D. L. (2006). When the Red Sox shocked the Yankees: Comparing negative and positive memories. Psychonomic Bulletin \& Review, 13(5), 757763. https://doi.org/10.3758/BF03193993

Kensinger, E. A., \& Ford, J. H. (2020). Retrieval of Emotional Events from Memory. Annual Review of Psychology, 71, 251-272. https://doi.org/10.1146/annurev-psych-010419$\underline{051123}$

Kensinger, E. A., \& Schacter, D. L. (2006). When the Red Sox shocked the Yankees: Comparing negative and positive memories. Psychonomic Bulletin \& Review, 13(5), 757-763. https://doi.org/10.3758/bf03193993

LaBar, K. S., \& Cabeza, R. (2006). Cognitive neuroscience of emotional memory. Nature Reviews Neuroscience, 7(1), 54-64. https://doi.org/10.1038/nrn1825

Laney, C., Campbell, H. V., Heuer, F., \& Reisberg, D. (2004). Memory for thematically arousing events. Memory and Cognition, 32(7), 1149-1159. https://doi.org/10.3758/BF03196888

Levine, L., \& Bluck, S. (2004). Painting with broad strokes: Happiness and the malleability of event memory. Cognition \& Emotion, 18(4), 559-574. https://doi.org/10.1080/02699930341000446 
Levine, L. J., \& Edelstein, R. S. (2010). Emotion and memory narrowing: A review and goalrelevance approach. (pp. 178-220). Psychology Press.

Levine, B., Svoboda, E., Hay, J. F., Winocur, G., \& Moscovitch, M. (2002). Aging and autobiographical memory: Dissociating episodic from semantic retrieval. Psychology and Aging, 17(4), 677. https://doi.org/10.1037/0882-7974.17.4.677

Loftus, E. F., Loftus, G. R., \& Messo, J. (1987). Some facts about "weapon focus". Law and human behavior, 11(1), 55-62. https://doi.org/10.1007/BF01044839

Mackay, D. G., Shafto, M., Taylor, J. K., Marian, D. E., Abrams, L., \& Dyer, J. R. (2004).

Relations between emotion, memory, and attention: Evidence from taboo stroop, lexical decision, and immediate memory tasks. Memory \& Cognition, 32(3), 474-488. https://doi.org/10.3758/BF03195840

Madan, C. R., Caplan, J. B., Lau, C. S., \& Fujiwara, E. (2012). Emotional arousal does not enhance association-memory. Journal of Memory and Language, 66(4), 695-716. https://doi.org/10.1016/j.jml.2012.04.001

Madan, C. R., Scott, S., \& Kensinger, E. A. (2019). Positive emotion enhances associationmemory. Emotion (Washington, D.C.), 19(4), 733-740. https://doi.org/10.1037/emo0000465

Maddock, R. J., \& Frein, S. T. (2009). Reduced memory for the spatial and temporal context of unpleasant words. Cognition and Emotion, 23(1), 96-117. https://doi.org/10.1080/02699930801948977

Makowski, D., Sperduti, M., Nicolas, S., \& Piolino, P. (2017). "Being there" and remembering it: Presence improves memory encoding. Consciousness and Cognition, 53, 194-202. https://doi.org/10.1016/j.concog.2017.06.015

Marchewka, A., Zurawski, Ł., Jednoróg, K., \& Grabowska, A. (2014). The Nencki Affective Picture System (NAPS): introduction to a novel, standardized, wide-range, high-quality, 
realistic picture database. Behavior research methods, 46(2), 596-610. https://doi.org/10.3758/s13428-013-0379-1

Mather, M. (2007). Emotional arousal and memory binding: An object-based framework. Perspectives on Psychological Science, 2(1), 33-52. https://doi.org/10.1111/j.1745$\underline{6916.2007 .00028 . x}$

Mather, M., \& Sutherland, M. R. (2011). Arousal-biased competition in perception and memory. Perspectives on Psychological Science, 6(2), 114-133. https://doi.org/10.1177/1745691611400234

McKinnon, M. C., Palombo, D. J., Nazarov, A., Kumar, N., Khuu, W., \& Levine, B. (2015). Threat of death and autobiographical memory: A study of passengers from Flight AT236. Clinical Psychological Science, 3(4), 487-502. https://doi.org/10.1177/2167702614542280

Murty, V. P., \& Adcock, R. A. (2017). Distinct medial temporal lobe network states as neural contexts for motivated memory formation. In D. E. Hannula \& M. C. Duff (Eds.), The hippocampus from cells to systems: Structure, connectivity, and functional contributions to memory and flexible cognition (pp. 467-501). Springer International Publishing. https://doi.org/10.1007/978-3-319-50406-3 15

Orbach, Y., Lamb, M. E., La Rooy, D., \& Pipe, M. E. (2012). A case study of witness consistency and memory recovery across multiple investigative interviews. Applied Cognitive Psychology, 26(1), 118-129. https://doi.org/10.1002/acp.1803

Palombo, D. J., \& Cocquyt, C. (2020). Emotion in context: Remembering when. Trends in Cognitive Sciences, 24(9), 687-690. https://doi:10.1016/j.tics.2020.05.017

Palombo, D. J., Te, A. A., Checknita, K. J., \& Madan, C. R. (2021). Exploring the Facets of Emotional Episodic Memory: Remembering "What," "When," and "Which." Psychological Science, 32(7), 1104-1114. https://doi.org/10.1177/0956797621991548 
Petrucci, A. S., \& Palombo, D. J. (2021). A matter of time: How does emotion influence temporal aspects of remembering? Cognition and Emotion, 35(8), 1499-1515. https://doi.org/10.1080/02699931.2021.1976733

Polyn, S. M., Norman, K. A., \& Kahana, M. J. (2009). A context maintenance and retrieval model of organizational processes in free recall. Psychological Review, 116(1), 129. https://doi.org/10.1037/a0014420

Rimmele, U., Davachi, L., Petrov, R., Dougal, S., \& Phelps, E. A. (2011). Emotion enhances the subjective feeling of remembering, despite lower accuracy for contextual details. Emotion, 11(3), 553-562. https://doi.org/10.1037/a0024246

Rimmele, U., Davachi, L., \& Phelps, E. A. (2012). Memory for time and place contributes to enhanced confidence in memories for emotional events. Emotion, 12(4), 834-846. https://doi.org/10.1037/a0028003

Rivera, J. (Producer), \& Docter, P. (Director). (2009). Up [Motion picture]. United States: Walt Disney Pictures Pixar Animation Studios.

Schmidt, K., Patnaik, P., \& Kensinger, E. A. (2011). Emotion's influence on memory for spatial and temporal context. Cognition \& Emotion, 25(2), 229-243. https://doi.org/10.1080/02699931.2010.483123

Screwvala, R., Roy Kapur, S., Jindal, S., (Producers), \& Kapri, V. (Director). (2018). Pihu [Motion Picture]. India: Roy Kapur Films.

Talmi, D., Anderson, A. K., Riggs, L., Caplan, J. B., \& Moscovitch, M. (2008). Immediate memory consequences of the effect of emotion on attention to pictures. Learning \& Memory, 15(3), 172-182. https://doi.org/10.1101//m.722908

Talmi, D., Lohnas, L. J., \& Daw, N. D. (2019). A retrieved context model of the emotional modulation of memory. Psychological Review, 126(4), 455-485. https://doi.org/10.1037/rev0000132 
Talmi, D., Moscovitch, M. (2004). Can semantic relatedness explain the enhancement of memory for emotional words? Memory \& Cognition, 32, 742-751. https://doi.org/10.3758/BF03195864

Todd, R. M., Miskovic, V., Chikazoe, J., \& Anderson, A. K. (2020). Emotional objectivity: Neural representations of emotions and their interaction with cognition. Annual Review of Psychology, 71(1), 25-48. https://doi.org/10.1146/annurev-psych-010419-051044

Tsikandilakis, M., Bali, P., Derrfuss, J., \& Chapman, P. (2019). Anger and hostility: Are they different? An analytical exploration of facial-expressive differences, and physiological and facial-emotional responses. Cognition and Emotion, 34(3), 581-595. https://doi.org/10.1080/02699931.2019.1664415

Tulving, E. (1972). Episodic and semantic memory. In E. Tulving \& W. Donaldson (Eds.). Organization of memory (pp. 381-403). New York, NY: Academic Press.

Wardell, V., Esposito, C. L., Madan, C. R., \& Palombo, D. J. (2021). Semi-automated transcription and scoring of autobiographical memory narratives. Behavior Research Methods, 53(2), 507-517. https://doi.org/10.3758/s13428-020-01437-w 


\section{Author Contributions}

Design (all), data collection (D.K.D.), analyses (all except A.S.P.), writing (D.K.D. and V.W. with contributions from all).

\section{Acknowledgements}

The authors thank Nick Diamond for discussion and Taylyn Jameson for research assistance. We would also like to thank Rajesh Manocha for helping us purchase a DVD copy of the movie Pihu. D.J.P is supported by an NSERC Discovery Grant (RGPIN-2019-04596) and the John R. Evans Leaders Fund from the Canadian Foundation for Innovation (38817). V.W. is supported by an NSERC CGS-M. K.C. is supported by an NSERC USRA. The authors declare no conflicts of interest. 


\section{Supplemental Material}

These online supplemental materials provide an outline of procedures we followed to run our analyses that are not reported in the Main text. These materials have been broken down into four sections: study stimuli, norming study, auxiliary materials and methods, and additional analyses. The additional analyses explore (1) arousal and valence ratings of the video stimuli between Hindi and non-Hindi speakers, (2) alternative approaches used to compute temporal order scores for the temporal-order reconstruction task, and (3) duration memory.

\section{Study Stimuli}

Table S1 provides a brief description of each clip in the high- and low-emotion videos as well as timestamps for each $20 \mathrm{sec}$ long clip in the original Pihu film (Screwvala et al., 2018).

\section{Table S1}

\section{Beginning Bookend Clips}

\begin{tabular}{|c|c|c|c|c|}
\hline Clip 1 & $04: 40$ & \multicolumn{3}{|l|}{ Pihu wakes up in bed } \\
\hline Clip 2 & 07:01 & \multicolumn{3}{|l|}{ Pihu explores the living room } \\
\hline Clip 3 & $14: 29$ & \multicolumn{3}{|c|}{ Pihu carries a chair up the stairs } \\
\hline \multicolumn{5}{|c|}{ Middle Clips } \\
\hline & & High-Emotion & & Low-Emotion \\
\hline Clip 4 & $17: 20$ & $\begin{array}{l}\text { Pihu climbs on top of dead } \\
\text { mother and cries; bottle of } \\
\text { pills falls from mother's } \\
\text { hand }\end{array}$ & $35: 13$ & $\begin{array}{l}\text { Pihu eats a piece of chocolate } \\
\text { cake from a paper plate while } \\
\text { sitting on the ground }\end{array}$ \\
\hline Clip 5 & 41:04 & $\begin{array}{c}\text { Pihu plays with balloons } \\
\text { near a hot iron; a balloon } \\
\text { pops }\end{array}$ & $37: 55$ & $\begin{array}{l}\text { Pihu eats bread and jam while } \\
\text { surrounded by a mess of food } \\
\text { items from the refrigerator and } \\
\text { with jam all over her mouth }\end{array}$ \\
\hline Clip 6 & $30: 43$ & $\begin{array}{l}\text { Pihu microwaves chapati } \\
\text { and burns her hand on it }\end{array}$ & 1:00:39 & $\begin{array}{c}\text { Pihu goes through a photo } \\
\text { album }\end{array}$ \\
\hline Clip 7 & 07:58 & $\begin{array}{l}\text { Pihu tiptoes on a chair to } \\
\text { reach the running tap; } \\
\text { hears sudden loud bang }\end{array}$ & 01:12:39 & $\begin{array}{l}\text { Pihu speaks on the phone with } \\
\text { man and laughs }\end{array}$ \\
\hline Clip 8 & $50: 11$ & $\begin{array}{l}\text { Pihu cries heavily while } \\
\text { walking down the stairs }\end{array}$ & $57: 58$ & $\begin{array}{l}\text { Pihu gets excited after seeing } \\
\text { a plane }\end{array}$ \\
\hline
\end{tabular}




\begin{tabular}{|c|c|c|c|c|}
\hline Clip 9 & 55:05 & $\begin{array}{l}\text { Pihu tries to grab her doll } \\
\text { that fell over the balcony } \\
\text { ledge }\end{array}$ & $29: 18$ & $\begin{array}{l}\text { Pihu wipes spilt milk off the } \\
\text { floor with a rag }\end{array}$ \\
\hline Clip 10 & 22:36 & $\begin{array}{l}\text { Pihu plays with makeup in } \\
\text { front of a mirror with a } \\
\text { (possible) suicide note }\end{array}$ & 08:51 & $\begin{array}{c}\text { Pihu changes TV channel and } \\
\text { dances to music }\end{array}$ \\
\hline Clip 11 & 01:02:22 & $\begin{array}{l}\text { Man calls Pihu on the } \\
\text { phone and tries to reach } \\
\text { her mother }\end{array}$ & 01:10:12 & $\begin{array}{l}\text { Pihu eats under the table and } \\
\text { shifts attention to knocking on } \\
\text { door }\end{array}$ \\
\hline Clip 12 & $36: 28$ & $\begin{array}{l}\text { Pihu traps herself in the } \\
\text { refrigerator }\end{array}$ & 13:26 & $\begin{array}{l}\text { Pihu gets on the toilet and } \\
\text { urinates }\end{array}$ \\
\hline Clip 13 & 45:31 & $\begin{array}{l}\text { Pihu tampers with } \\
\text { electrical wires and open } \\
\text { socket }\end{array}$ & 47:35 & $\begin{array}{l}\text { Pihu takes a nap on the living } \\
\text { room floor }\end{array}$ \\
\hline Clip 14 & 33:14 & $\begin{array}{l}\text { Pihu places a chapati on } \\
\text { the stove with open flame }\end{array}$ & 16:01 & Pihu brushes her teeth \\
\hline Clip 15 & $26: 17$ & $\begin{array}{l}\text { Burning piece of cloth falls } \\
\text { to the ground from ironing } \\
\text { board; Pihu sits with it }\end{array}$ & $20: 36$ & $\begin{array}{l}\text { Pihu reaches for phone stuck } \\
\text { in space under shelf }\end{array}$ \\
\hline Clip 16 & $01: 06: 22$ & $\begin{array}{l}\text { Pihu pours bleach into her } \\
\text { milk bottle }\end{array}$ & 23:46 & $\begin{array}{c}\text { Pihu looks through cabinet } \\
\text { drawers }\end{array}$ \\
\hline Clip 17 & 20:00 & $\begin{array}{l}\text { Pihu climbs onto unsteady } \\
\text { stool to reach ringing } \\
\text { phone }\end{array}$ & 06:24 & Pihu walks down flight of stairs \\
\hline Clip 18 & 43:56 & $\begin{array}{l}\text { Pihu tugs on phone wire } \\
\text { wrapped around iron board } \\
\text { with steaming iron on it }\end{array}$ & $52: 55$ & $\begin{array}{l}\text { Pihu plays around on the } \\
\text { balcony with her toy doll }\end{array}$ \\
\hline Clip 19 & 48:47 & $\begin{array}{c}\text { Electrical explosion in } \\
\text { kitchen; Pihu watches and } \\
\text { cries }\end{array}$ & $59: 48$ & $\begin{array}{l}\text { Pihu plays in her toy tent with } \\
\text { a ball and a baby doll }\end{array}$ \\
\hline \multicolumn{5}{|c|}{ End Bookend Clips } \\
\hline Clip 20 & 01:03:39 & \multicolumn{3}{|c|}{ Pihu plays around, dancing and rolling on the ground } \\
\hline Clip 21 & 15:34 & \multicolumn{3}{|c|}{ Pihu plays with a bidet in the toilet } \\
\hline Clip 22 & $34: 43$ & \multicolumn{3}{|c|}{ Pihu takes a slice of cake out of the refrigerator and eats it } \\
\hline
\end{tabular}

\section{Norming Study}

Participants. A total of 194 undergraduate students at the University of British Columbia registered for the norming study in exchange for course credit. Of the 194, 30 participants were excluded as they either did not complete the study in its entirety or opted out of participating due 
to trigger warnings. A further four participants were excluded because they had seen the film prior to participating in the study, leaving a final sample of 160 participants.

Procedure. Participants were randomly assigned to either a high-emotion or lowemotion condition (see main text). They were presented with each one of the 22 clips from the assigned video for their condition in separate pages, in the order that they appeared in the video. As in the main study, the videos from both emotion conditions contained the same six bookend clips as well as 16 middle clips that were each unique to the emotion condition. After watching each $20 \mathrm{sec}$ clip, participants were tasked to rate the arousal and valence of the clip they had watched. Arousal was rated on a nine-point scale $(1=$ little to no intensity, $5=$ moderate intensity, $9=$ extreme intensity) as was valence $(1=$ very negative, $5=$ neutral, $9=$ very positive). At the end of the study, participants were asked if they were familiar with the film prior to their participation and if they understood Hindi, after which they were debriefed.

Data Analysis. Averaged arousal and valence ratings (i.e., averaging across individual clips) for bookend and middle clips were calculated for each participant. Mann-Whitney $\mathrm{U}$ tests were then performed to compare ratings for bookend and middle clips across emotion conditions. We then compared ratings within emotion conditions across non-Hindi and Hindi speakers, as there was Hindi dialogue in the videos, albeit very minimal.

Results. The arousal rating for the middle clips was greater in the high-emotion $(M d n=5.06)$ than the low-emotion condition $(M d n=3.12 ; U=5026.50, Z=-6.23, p<.001$; Figure S1). The arousal rating for the bookend clips did not statistically differ between the highemotion $(M d n=2.67)$ and low-emotion conditions $(M d n=2.67 ; U=3395.00, Z=-0.67, p=$ .505 ; Figure S1).

The valence rating for the middle clips was more negative in the high-emotion condition $(M d n=3.06)$ than the low-emotion condition $(M d n=4.44 ; U=800.00, Z=-8.19, p<.001$; Figure S1). The valence rating for the bookend clips did not statistically differ between the highemotion $(M d n=4.83)$ and low-emotion conditions $(M d n=4.67 ; \mathrm{U}=3449.00, \mathrm{Z}=-0.85$, 
$p=.393 ;$ Figure S1). These results validated the use of the high- and low-emotion videos for use in the main study.

\section{Figure S1}

Norming Study - Arousal and Valence Ratings by Condition for Video Clips

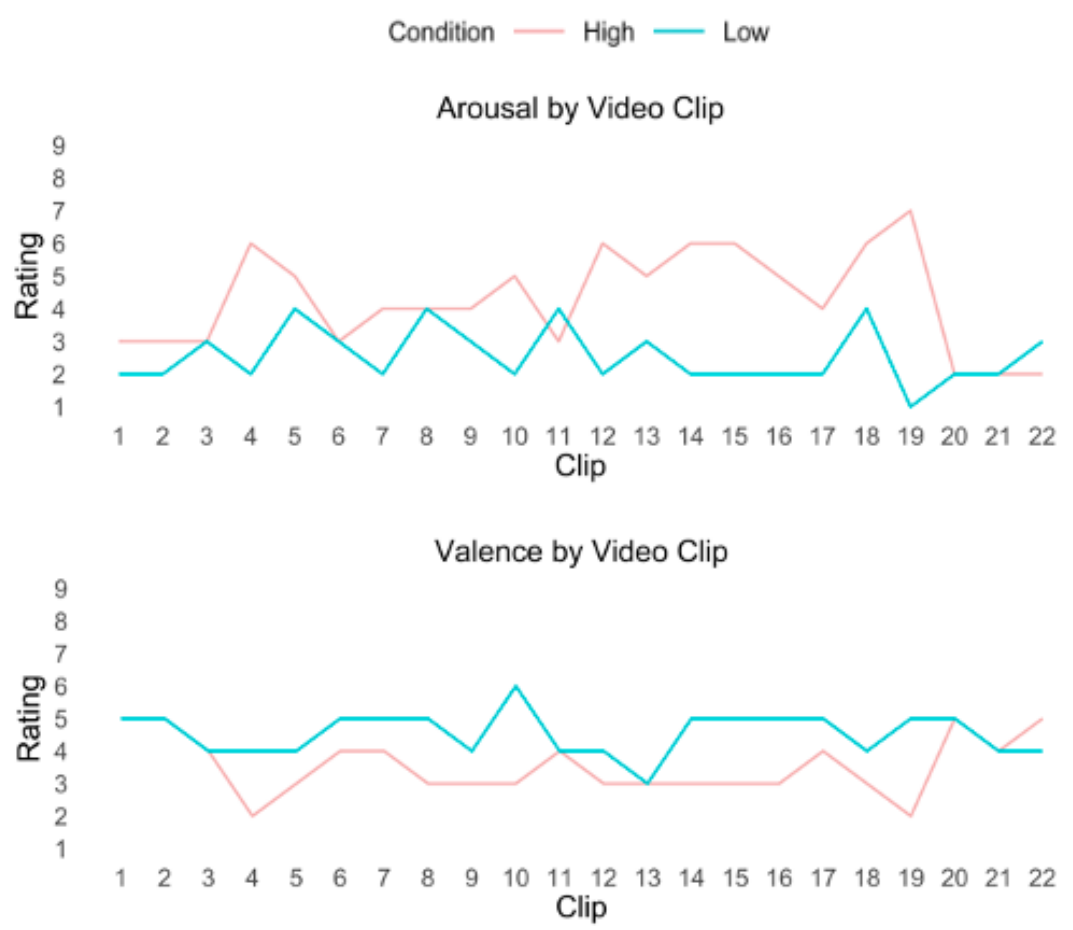

Note. Arousal (upper) and valence (lower) ratings for individual clips across high- and lowemotion video conditions. Clips 1-3 and 20-22 represent bookend clips, clips 4-19 represent middle clips. The line intersects the median rating for each clip. This figure is for display purposes only (to provide more fine-grained, clip-by-clip information).

We then compared ratings within emotion conditions between Hindi and non-Hindi speakers to ensure that knowledge of Hindi did not affect the ratings, as there was some Hindi dialogue in the videos (see Table S2). In the high-emotion condition, there was no difference observed between arousal ratings made by non-Hindi and Hindi speakers for middle 
$(U=327.00, Z=-0.18, p=.859)$, or bookend clips $(U=248.50, Z=-1.02, p=.308)$. Further, there was no difference in valence ratings of non-Hindi and Hindi speakers for middle $(U=311.50, Z=-0.05, p=.963)$, or bookend clips $(U=292.00, Z=-0.35, p=.728)$. Similarly, in the low-emotion condition, there was no difference observed between arousal ratings made by non-Hindi and Hindi speakers for middle clips $(U=276.50, Z=-0.29, p=.775)$, or bookend clips $(U=273.50, Z=-0.24, p=.814)$. Further, there was no difference in valence ratings of non-Hindi and Hindi speakers for middle $(U=241.50, Z=-0.29, p=.775)$, or bookend clips $(U=266.50, Z=-0.12, p=.906)$.

\section{Table S2}

\begin{tabular}{|c|c|c|c|c|c|}
\hline \multicolumn{6}{|c|}{ High-Emotion } \\
\hline & & \multicolumn{2}{|c|}{ Middle Clips } & \multicolumn{2}{|c|}{ Bookend Clips } \\
\hline & & Arousal & Valence & Arousal & Valence \\
\hline & $N$ & Mdn & $M d n$ & Mdn & $M d n$ \\
\hline Hindi Speakers & 9 & 5.25 & 2.63 & 2.00 & 4.33 \\
\hline Non-Hindi Speakers & 70 & 5.03 & 3.06 & 2.83 & 4.83 \\
\hline \multicolumn{6}{|c|}{ Low-Emotion } \\
\hline & & \multicolumn{2}{|c|}{ Middle Clips } & \multicolumn{2}{|c|}{ Bookend Clips } \\
\hline & & Arousal & Valence & Arousal & Valence \\
\hline & $N$ & Mdn & $M d n$ & $M d n$ & $M d n$ \\
\hline Hindi Speakers & 7 & 3.44 & 4.31 & 2.83 & 4.67 \\
\hline Non-Hindi Speakers & 74 & 3.03 & 4.47 & 2.67 & 4.67 \\
\hline
\end{tabular}

\section{Auxiliary Materials and Methods}

Gender Distribution. Table S3 presents a breakdown of the gender distribution of participants in the main study.

\section{Table S3}

Gender Distribution 


\begin{tabular}{lcccccc}
\cline { 2 - 6 } & $N$ & $\%$ & $N$ & $\%$ & $N$ & $\%$ \\
\hline Cisgender, man & 17 & 6.16 & 10 & 7.52 & 7 & 4.90 \\
Cisgender, woman & 76 & 27.54 & 37 & 27.82 & 39 & 27.27 \\
Cisgender, non-binary & 1 & 0.36 & 0 & 0 & 1 & 0.70 \\
Cisgender, unspecified & 42 & 15.22 & 15 & 11.28 & 27 & 18.88 \\
Unspecified, man & 23 & 8.33 & 13 & 9.77 & 10 & 6.99 \\
Unspecified, woman & 110 & 39.86 & 55 & 41.35 & 55 & 38.46 \\
Unspecified, non-binary & 1 & 0.36 & 0 & 0 & 1 & 0.70 \\
Unspecified, non-binary, woman & 2 & 0.72 & 1 & 0.75 & 1 & 0.70 \\
Preferred not to answer & 4 & 1.45 & 2 & 1.50 & 2 & 1.40 \\
\hline
\end{tabular}

Free Recall Instructions. For the main study, prior to completing the free recall task, participants were provided with the following descriptions of the first scene in the Disney Pixar film Up (Rivera \& Docter, 2009). This was provided to ensure participants understood the types of details we were requesting them to provide.

"First I saw a young boy with glasses and a young girl lying stomach down together on a bed and reading a book. It was nighttime but they had a flashlight which they shined in a fort they built with blankets. The girl was talking about the book animatedly but the boy did not say anything and looked a bit nervous. The girl then left the room by climbing out of the window and the boy followed, watching her through the window dreamily. Then the scene changed to a photograph being taken of a couple on their wedding day - the man was wearing the same glasses as the boy in the earlier scene. The woman kissed the man excitedly and all other details explained in a similar manner."

Free Recall Detail Accuracy Scoring. Below we provide examples of free recall narratives from the high-emotion condition, one that had a low number of details (Example A) 
and one with a high number of details (Example B; see Figure S2). These examples are provided to give the reader a sense of how the scoring was completed.

\section{Figure S2}

\section{Example A}

Narrative Recall:

The video started with a toddler/CONS/ opening her eyes /CONS/. She found her mom /CONS/ who passed away /CONS/ holding a bottle of pills /CONS/. The little girl was crying/CONS/. Later on, she performed several tasks/CONS/ including warming up her food/CONS/ in a microwave/CONS/, talking to her father /CONS/ and ironing /CONS/. At one point she also put herself in the fridge /CONS/ and also ate cake /CONS/ from the fridge /CONS/.

[ER-2]

Example B 


\begin{abstract}
Narrative Recall:
A little South Asian /CONS/ girl /CONS/ woke up /CONS/. Her appearance is disheveled /CONS/ - she has a blue shirt/CONS/, messy hair/CONS/, and party style hair clip /CONS/ that are hanging loosely in her hair /CONS/. She seemed relaxed and carefree at first/CONS/. She pushed this stool thing /CONS/ up the stairs /CONS/ with great effort/CONS/. Then the scene cut to her entering her mom's room /CONS/ and crying/CONS/, yelling mama! /CONS/ Her mom /CONS/ overdosed on some pills /CONS/. Then the girl was playing around /CONS/ in the mom's room /CONS/ and there were balloons everywhere /CONS/. The girl doesn't seem to understand her mom passed away/CONS/. There was a iron /CONS/ on an ironing board/CONS/ and that looked like it might fall on her /CONS/. Then she has the stool thing/CONS/ at the sink/CONS/ and she tries to wash her hands /CONS/ but has trouble reaching it /CONS/. Then there was a scene in the mom's bedroom /CONS/ where the pills /CONS/ were scattered /CONS/ on the floor/CONS/ and it seems like the mom wrote a suicide note /CONS/ in red lipstick/CONS/. The last word said Goodbye /CONS/. The girl was playing with the lipstick/CONS/. The girl was trying to reach a doll/CONS/ stuck on the ledge of the balcony /CONS/ with her foot/CONS/, but it feel off the ledge /CONS/. She seemed shocked and disappointed it fell/CONS/. The girl tried to cook some bread /CONS/ next on the stove/CONS/, but it was burned/CONS/. Then the girl was playing with the refrigerator/CONS/. She finally shut the door on the fridge/CONS/ with her inside /CONS/. Then the scene cut so that she was in a tight place /CONS/ with a bunch of exposed wires /CONS/. She plugged something inside /COM/ and some Christmas lights /CONS/ in the kitchen lit up /CONS/, hummed/CONS/, and then exploded/CONS/. The girl started to cry /CONS/ while calling for her mom /CONS/. I think in the next scene she also tried to wash some blue /CONS/ slippers (flip flops)/CONS/ with a bidet /CONS/ and told her mom she cleaned them /CONS/. I think the next scene was the girl playing in the mom's room /CONS/ with a phone/CONS/. There was a real phone that kept ringing /CONS/, but the girl didn't really answer it /CONS/ and played with her toy phone instead /CONS/. The cord from the toy phone got tangled /CONS/ with the ironing board /CONS/ and there was a loud crash/CONS/, so I assume the iron fell/OTH/. In the final scene, the real phone kept ringing again /COM/. but the girl ignored it and ate some chocolate looking cake /CONS/ from the fridge /CONS/.

[ER-6]
\end{abstract}

Note. Example of high-emotion condition free recalls of $(A)$ a minimally detailed recall and

(B) a highly detailed recall. Episodic richness (ER) ratings are noted at the end of the

transcript. CONS=consistent; $\mathrm{COM}=$ error of commission;

OTH=metacognition/editorialization. 
Free Recall Temporal Clustering Scoring. Below we provide an example calculation of the temporal clustering score for a free recall narrative (see Figure S3).

\section{Figure S3}

Example A

\begin{tabular}{l} 
1) Score transcripts for middle and bookend clips \\
$\begin{array}{l}\text { Narrative Recall: } \\
\text { The video started with a toddler opening her eyes } / 1 / \text {. She found her mom who passed away holding a bottle of } \\
\text { pills. The little girl was crying } / 4 / \text {. Later on, she performed several tasks including warming up her food in a } \\
\text { microwave } / 6 / \text {, talking to her father } / 11 / \text { and ironing } / 15 / \text {. At one point she also put herself in the fridge } / 12 / \\
\text { and also ate cake from the fridge } / 22 / \text {. }\end{array}$ \\
\hline
\end{tabular}

2) Identify clips
\begin{tabular}{|c|c|c|}
\hline $4 \rightarrow 6 \rightarrow 11 \rightarrow 15 \rightarrow 12$ & $\begin{array}{c}4 \rightarrow 6 \rightarrow 11 \rightarrow 15 \rightarrow 12 \\
\text { Remove duplicates }\end{array}$ \\
Identify middle clips & Recode clip numbers to start at 1 \\
\hline
\end{tabular}

3) Calculate transition score for each transition

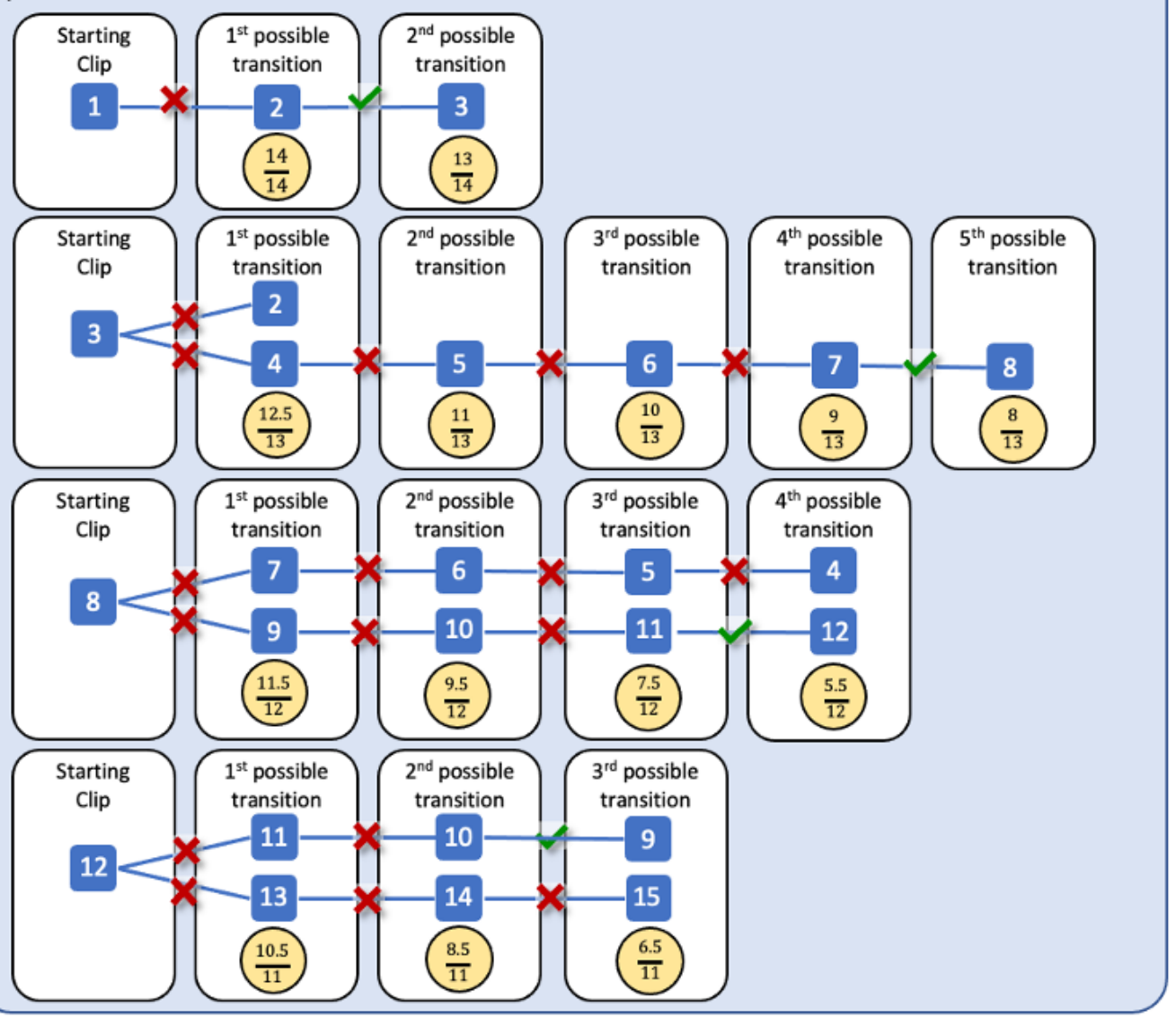

4) Average transition scores to calculate temporal cluster

$$
\frac{\left(\frac{13}{14}\right)+\left(\frac{8}{13}\right)+\left(\frac{5.5}{12}\right)+\left(\frac{6.5}{11}\right)}{4}=0.6483
$$


Example B

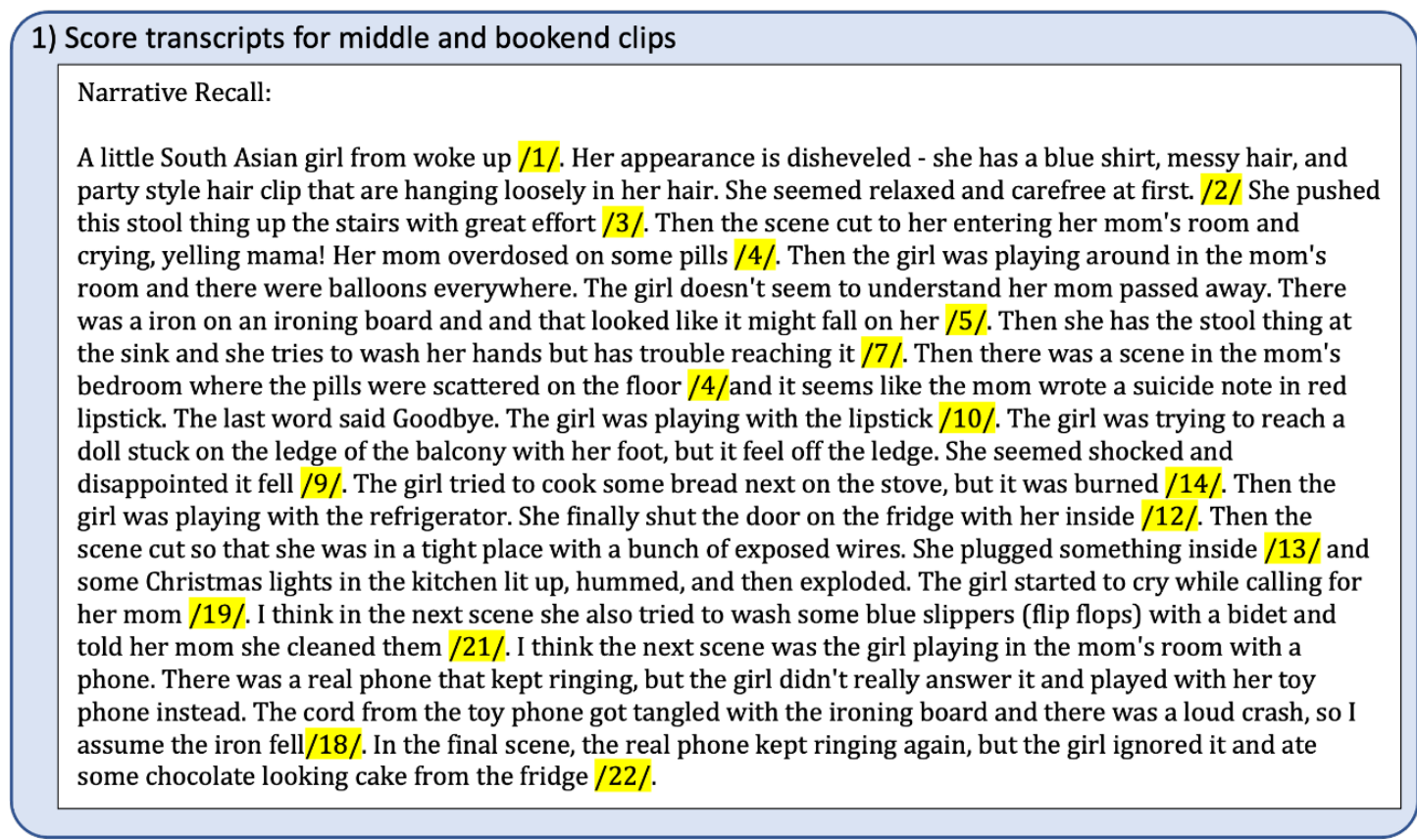

\section{2) Identify clips}

$4 \rightarrow 5 \rightarrow 7 \rightarrow 4 \rightarrow 10 \rightarrow 9 \rightarrow 14 \rightarrow 12 \rightarrow 13 \rightarrow 19 \rightarrow 18$

Identify middle clips

$4 \rightarrow 5 \rightarrow 7 \rightarrow 10 \rightarrow 9 \rightarrow 14 \rightarrow 12 \rightarrow 13 \rightarrow 19 \rightarrow 18$ Remove duplicates

$1 \rightarrow 2 \rightarrow 4 \rightarrow 7 \rightarrow 6 \rightarrow 11 \rightarrow 9 \rightarrow 10 \rightarrow 16 \rightarrow 15$

Recode clip numbers to start at 1

\section{3) Calculate transition score for each transition}

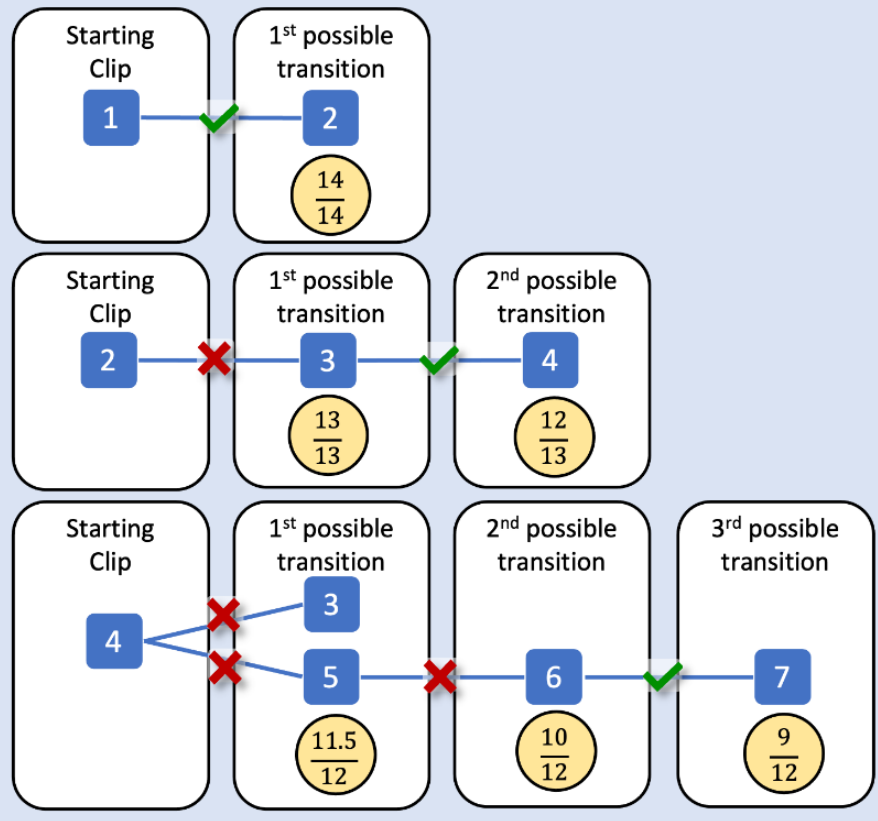




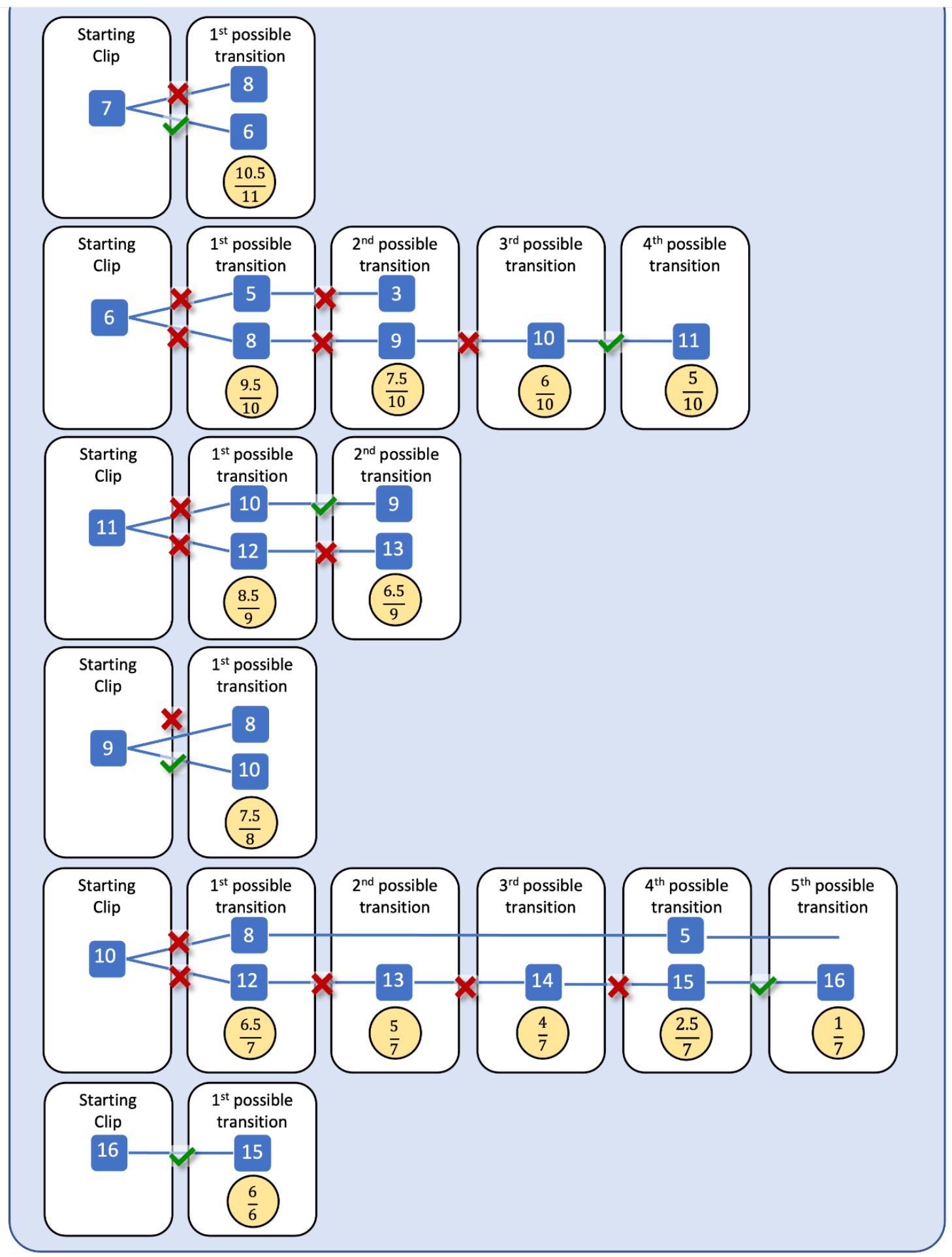

\section{4) Average transition scores to calculate temporal cluster}

$$
\frac{\left(\frac{14}{14}\right)+\left(\frac{12}{13}\right)+\left(\frac{9}{12}\right)+\left(\frac{10.5}{11}\right)+\left(\frac{5}{10}\right)+\left(\frac{6.5}{9}\right)+\left(\frac{7.5}{8}\right)+\left(\frac{1}{7}\right)+\left(\frac{6}{6}\right)}{9}=0.7700
$$


Note. Two examples of temporal order clustering calculation. Highlighted numbers correspond to the clip number identified in the narrative. Bookend clips were removed from the temporal clustering analysis, leaving only the middle, condition-dependent clips to be part of the clustering score. With 16 clips, the total range of clips was 15 . In order for the calculation of the furthest transition to have a score of 0 , counts were done using zerobased indexes (i.e., 14/14 for the closest transition and 0/14 for the furthest transition). Only the first mention of each clip was included, that is, repetitions of clips were removed. As such, once a clip was mentioned it was no longer a 'possible transition'.

Free Recall Reliability. To ensure scoring of the free recall narratives was reliable between the two scorers, we present Cronbach's alpha scores for the detail types scored. A two-way intraclass correlation (ICC) showed excellent agreement between the primary and secondary scorers (see Table S4). Furthermore, as we could not blind scorers to condition due to the nature of our stimuli, we compared the word count of recalls for a more objective, albeit less nuanced measure of detail production. Recalls from the high-emotion condition $(M d n=$ 186) contained more words than the low-emotion condition $(M d n=154 ; U=11352.00$, $Z=-2.78, p=.005)$.

\section{Table S4}

Cronbach's Alpha Scores for Individual Detail Types

\begin{tabular}{lrll}
\hline & ICC & Fixed Effects & $p$ \\
\hline \multicolumn{1}{c}{ Across Emotion Conditions } & & \\
Total Details & .99 & $F(29,29)=67$ & $<.0001$ \\
Internal Details & .99 & $F(29,29)=81$ & $<.0001$ \\
Consistent Details & .98 & $F(29,29)=56$ & $<.0001$ \\
Episodic Richness & .82 & $F(29,29)=5.6$ & $<.0001$ \\
\hline \multicolumn{1}{c}{ High-Emotion Condition } & & \\
\hline Total Details & .98 & $F(14,14)=54$ & $<.0001$ \\
Internal Details & .99 & $F(14,14)=75$ & $<.0001$
\end{tabular}




\begin{tabular}{lcll} 
Consistent Details & .98 & $F(14,14)=57$ & $<.0001$ \\
Episodic Richness & .83 & $F(14,14)=5.9$ & .0011 \\
\hline \multicolumn{1}{c}{ Low-Emotion Condition } & & & \\
\hline Total Details & .99 & $F(14,14)=147$ & $<.0001$ \\
Internal Details & .99 & $F(14,14)=87$ & $<.0001$ \\
Consistent Details & .98 & $F(14,14)=49$ & $<.0001$ \\
Episodic Richness & .85 & $F(14,14)=6.7$ & .0005
\end{tabular}

Note. Inter-rater reliability for consistency scoring, using a two-way ANOVA with raters as fixed effects. We note that scorers were unable to be blinded to emotional condition, given the depiction of condition-specific clips in recall. However, the second scorer was blind to the hypotheses of the study.

\section{Additional Analyses}

Hindi Speakers. For the main study, we also conducted a Mann-Whitney $U$ test to compare arousal and valence ratings between participants who understood Hindi versus those who did not, because there was some Hindi dialogue in the videos. This was done with the purpose of validating the inclusion of Hindi speakers in our analyses for the main study. In the high-emotion condition, no differences between Hindi and non-Hindi speakers were observed for arousal $(U=867.50, Z=-0.25, p=.800)$ and valence ratings $(U=980.00, Z=-1.12$, $p=.262)$ (Table S5). Similarly, in the low- emotion condition, no differences between Hindi and non-Hindi speakers were observed for arousal $(U=1048.00, Z=-1.44, p=.150)$, or valence ratings $(U=602.50, Z=-1.74, p=.082 ;$ Table S5).

\section{Table S5}

Arousal and Valence Ratings for Non-Hindi and Hindi Speakers

\begin{tabular}{lccc}
\hline \multicolumn{4}{c}{ High-Emotion } \\
& & Arousal & Valence \\
& $N$ & Mdn & Mdn \\
\hline Hindi Speakers & 14 & 7 & 2.5
\end{tabular}




\begin{tabular}{lccc} 
Non-Hindi Speakers & 119 & 7 & 2 \\
\hline & Low-Emotion & \\
\hline & $N$ & Mrousal & Valence \\
\hline Hindi Speakers & 13 & 6 & Mdn \\
Non-Hindi Speakers & 130 & 5 & 3 \\
\hline
\end{tabular}

Alternative Approaches to Compute Temporal Order Scores for the Temporal-

Order Reconstruction Task. To analyze the output from the temporal-order reconstruction task, we computed relative and absolute order scores for each participant in addition to Spearman correlation scores reported in the Main paper. The relative order score was derived as the difference between the participant's recalled rank of a respective scene and the subsequent ranks that it was correctly placed before, which was computed out of a score of 28 for each block (of eight items each, hence the total score is calculated with $7+6+5+4+3+2+1=$ 28). For example, if the participant ranked the clips in the following order of $1,12,7,3,6,10,11$, 15 , we calculate the relative order score with $7+1+3+4+3+2+1=21$. This is because clip 1 was correctly ranked before seven clips (clips $12,7,3,6,10,11,15$ ), clip 12 was correctly ranked before only one clip (clip 15), clip 7 was correctly ranked before three clips (clips 10, 11, 15), clip 3 was ranked correctly before four clips (clips $6,10,11,15$ ), clip 6 was ranked correctly before three clips (clips 10,11,15), clip 10 was ranked correctly before two clips (clips 11 and 15), and finally, clip 11 was ranked correctly before one clip (clip 15).

The absolute order score was computed as the average temporal error scores of each scene, wherein the absolute value of the difference between the correct ranking and the participant's recalled ranking of each scene was calculated and averaged.

There was a strong correlation between the average temporal error score and the Spearman correlation score across all blocks and conditions (high-emotion block $1=-0.90$; high-emotion block $2=-0.93 ;$ low-emotion block $1=-0.85$; low-emotion block $2=-0.86$ ). There 
was also a strong correlation found between the relative order score and the Spearman correlation score across all blocks and conditions (high-emotion block $1=0.93$; high-emotion block $2=0.95$; low-emotion block $1=0.86$; low-emotion block $2=0.87$ ). Due to the convergence of the scores, we focused only on Spearman correlations for our main analysis.

Duration Memory. As an exploratory analysis, a Mann-Whitney $\mathrm{U}$ test was conducted to determine if there was a difference in the remembered duration of the video (in minutes) between the low-emotion and high-emotion conditions. There was no significant difference between the low-emotion $(M d n=8.00)$ and high-emotion conditions $(M d n=10 ; U=9819.50$, $Z=0.48, p=.634)$. Moreover, a one-sample Mann-Whitney $U$ test showed that participants remembered the duration of the video as being longer than it was in both conditions (lowemotion: $U=6213, Z=2.15, p=.031$; high-emotion: $U=5339, Z=2.00, p=.046$ ). 